\title{
CD133 promotes resistance to trametinib in melanoma stem cells by activating an AKT / BCL-2 survival pathway
}

\author{
Cynthia M. Simbulan-Rosenthal', Yogameenakshi Haribabu', Sahar Vakili', Li-Wei Kuo1, Havens Clark1, Ryan \\ Dougherty1, Ryyan Alobaidi1, Bonnie Carney1, 2, Peter Sykora1,3 and Dean S. Rosenthal1*
}

\author{
1Department of Biochemistry and Molecular \& Cellular Biology, Georgetown University School of Medicine, \\ Washington, DC 20007, \\ ${ }^{2}$ MedStar Health Research Institute, Washington, D.C., \\ ${ }^{3}$ Amelia Technologies, Washington, DC 20007 \\ * Correspondence: Correspondence: rosenthd@georgetown.edu
}

\begin{abstract}
Malignant melanoma is a lethal skin cancer containing melanoma-initiating cells (MIC), implicated in tumorigenesis, invasion, and drug resistance, and characterized by elevated expression of stem cell markers, such as CD133. We previously showed that siRNA knockdown of CD133 enhances apoptosis induced by the MEK inhibitor trametinib in melanoma cells. The current study investigates underlying mechanisms of CD133's anti-apoptotic activity in patient-derived BAKP and POT cells, harboring difficult-to-treat $\mathrm{NRAS}^{\mathrm{Q} 61 \mathrm{~K}}$ and NRAS ${ }^{\mathrm{Q} 61 \mathrm{R}}$ drivers, after CRISPR-Cas9 CD133 knockdown or Dox-inducible expression of CD133. To maintain stable expression of CD133, MACS-sorted CD133(+) positive cells were expanded by ROCK-mediated conditional reprogramming of BAKP melanoma cells (BAKR). BAKR showed increased survival via reduced apoptosis after exposure to trametinib or DTIC, compared to BAKP. CRISPR-Cas9- mediated CD133 knockdown in BAKR cells (BAKR-T3) re-sensitized the cells, while CRISPR-Cas9 knockdown of CD133 in parental BAKP and POT cells even further increased trametinib-induced apoptosis (cleaved PARP) by reducing levels of anti-apoptotic BCL-xL, p-AKT, and p-BAD, and increasing pro-apoptotic BAD and active BAX. Dox-induced CD133 overexpression had the opposite effect, and blocked trametinib-induced apoptosis in both cell lines, coincident with elevated p-AKT, pBAD, BCL-2 and BCL-xL and decreased levels of the active form of BAX and caspases- 3 and -9 . The roles of CD133 in AKT and BAD phosphorylation, or in the upregulation of anti-apoptotic BCL-2 family members, was further investigated by AKT knockout with siRNA, or inhibition of BCL-2 family members with navitoclax (ABT-263). Similar to CD133 knockdown, AKT1/2 siRNA knockdown in BAKP cells also reduced p-BAD. CD133 knockdown (T3)-mediated reduction of pBAD levels was equivalent in AKT-knockdown or AKT control cells indicating that CD133 may be upstream of AKT signaling. In BAKP cells treated with trametinib and/or ABT-263, effects of ABT-263 mirrored CD133 knockdown, since levels of active BAX and cleaved-PARP in BAKP-SC (CD133-) cells increased to the same level as that exhibited by BAKP-T3 cells (CD133+). CD133 may therefore activate a survival pathway where 1) increased phosphorylation of AKT induces 2) phosphorylation and inactivation of $\mathrm{BAD}, 3$ ) decrease in the active form of $\mathrm{BAX}$, and 4) reduction in caspasemediated PARP cleavage, indicating apoptosis suppression leading to drug resistance in melanomas. Targeting survival pathways by which CD133 may confer chemoresistance in MICs can contribute to development of more effective treatments for patients with high-risk melanoma.
\end{abstract}

Keywords: melanoma initiating cells; CD133; drug resistance; apoptosis; caspase activation; CRISPR-Cas9 knockout; AKT; BAD; BCL-2 family

\section{Introduction}


Malignant melanoma remains a formidable challenge, with an estimated 106,110 new cases and 7,180 deaths in the United States in 2021, and a cure rate still below $10 \%$ for regional and distant stages [1]. Drug and immune resistance, together with invasion and metastasis, determine tumor progression, and ultimately, patient survival. With the highest mortality rate among skin cancers, cutaneous melanoma is associated with driver mutations in the MAPK, and other signaling pathways including RAS/PI3K/AKT, p16INK4a/CDK4/RB, and WNT and/or p53 [2]. Melanomagenesis is correlated with activating driver mutations primarily in codons V600 or K601 of BRAF ( $v$-raf; murine sarcoma viral oncogene homolog B1; 50\% of melanomas) or Q61 of NRAS (neuroblastoma RAS viral oncogene homolog; 20\%), amplification or activating mutations of C-KIT (2$8 \%$ ), or LOF mutations in the tumor suppressor NFI (nuclear factor I; $20 \%$ ).

The use of targeted kinase inhibitors, including BRAF and MEK inhibitors, combined with immune checkpoint inhibitors, has significantly improved progression-free and overall survival of melanoma patients [3,4]. Multiple FDA-approved kinase inhibitors currently in use for melanoma include a combination of the MEK inhibitor trametinib (GSK1120212), and BRAF inhibitor dabrafenib for BRAFV600 mutations. Unfortunately, many patients develop resistance, significantly reducing efficacy in response to these therapeutics $[5,6]$, a process accelerated by high mutation rates of cutaneous melanomas compared to other solid tumors $[7,8]$. Although $B R A F$-mutant melanoma is currently treatable with BRAF and MEK inhibitors in combination therapies, acquired resistance in $\sim 75 \%$ of melanomas can occur by reactivation of MAPK signaling after BRAF copy number gains and alternative splicing, or MEK1/2 and NRAS gain-of-function mutations; an additional $20 \%$ of BRAF inhibitor-resistant melanomas acquire mutations or upregulate compensatory PI3K/AKT survival pathways [9]. Although treatment for recalcitrant NRAS-mutant metastatic tumors has recently advanced for PDL1-expressing patients, with immunotherapies anti-PD-1, anti-PD-L1 and anti-CTLA4 proving efficacious in first-line treatments, a majority of patients remain unresponsive, as chemotherapy with dacarbazine (Dimethyl Triazeno Imidazol Carboxamide; DTIC), temozolomide, or carboplatin added as supplementary treatments show limited success [10].

Resistance to therapeutic combinations and melanoma recurrence have been attributed to subpopulations of "melanoma initiating cells" (MIC). These highly tumorigenic cancer stem cells are characterized by their ability to form melanospheres [11], and express receptors, cell adhesion molecules, or other stem cell markers. These include ABCB5 [12], CD133 [13], CD20 B lymphocyte antigen [14], CD44 [15], CD144/Vascular endothelial (VE)-cadherin [16], CD166/activated leukocyte cell adhesion molecule (ALCAM) [17], CD271/low affinity nerve growth factor receptor (LNGFR) [18], aldehyde dehydrogenase 1 (ALDH1) [19], Nestin [20], Tie1 [21], and JARID1B H3K4 demethylase [22]. Melanoma cells expressing CD133, ABCB5, and/or CD144 can form stem cell niches facilitating tumor blood supply [7]. We have also shown elevated expression of CD133 and ABCB5 in lymph node and distant organ metastasis in various stages of human cutaneous melanoma [13].

The $97 \mathrm{kDa}$ transmembrane glycoprotein CD133, also known as prominin1 (PROM1), is expressed in stem cells of normal neuronal and glial cells, hematopoietic cells, endothelial cells, and cells from adult kidney, mammary gland, salivary gland, placenta, trachea, testes, uterus, epidermis, and intestine [23-28]. CD133 is also expressed in cancer stem cells from multiple organs, including cancers of the brain, ovary, liver, prostate, pancreas, colon, in addition to melanomas [29-37]. Displaying stem cell properties of self-renewal and potency, cancer stem cells are assayed by serial propagation as tumors in immunocompromised mice [25, 32, 38]. However, the existence of melanoma stem cells can be model-specific [39], and melanomas possess microenvironment-regulated phenotypic plasticity [40-42]; thus, the less controversial term "melanoma-initiating cells" (MIC) is now used instead. Nonetheless, we, along with others, have shown that CD133(+) MIC are associated with drug resistance [43], as well as invasion and metastasis [43]. CD133overexpressing glioma [44], as well as melanoma cells [43] were determined to be drug- 
resistant, in part due to the induction of $\mathrm{ABCB} 1$ expression and activity. Because of these characteristics, CD133(+) MIC [13] and other cancer stem cells [45] have been shown to play critical roles in recurrence and reduced patient survival, and are of interest as an anticancer vaccination component, with some success in mouse models of melanoma [46].

We have shown that CD133 positivity is correlated with recurrent patient disease, poor clinical outcomes, and decreased overall patient survival. While CD133(+) cells isolated from these patients' tumors by magnetic sorting formed tumors in nude mouse xenografts, CD133(-) cells did not [13]. Resistance of CD133(+) and CD133(-) melanoma cells to MAPK inhibitors was examined by exposing separated CD133(+) and CD133(-) subpopulations to increasing concentrations of trametinib and/or dabrafenib. CD133(+) cells exhibited significantly higher IC50s for mono and dual MAPKI treatments. We showed a causal relationship between CD133 and drug resistance by siRNA knockdown in different melanoma cell lines, indicating that CD133 confers drug resistance in melanoma [43]. However, the mechanistic pathway leading from CD133 expression to increased chemoresistance has not been clarified, although it was suggested that alterations in levels or modification of apoptotic proteins (e.g., BCL-2 family members) by CD133-mediated pathways may represent a mechanism of resistance. Better delineation of the molecular mechanisms that control apoptosis is key to developing novel targets for therapeutic intervention in melanoma.

In the current study, we analyzed the potential mechanisms by which CD133 is involved in development of resistance against targeted kinase inhibitors such as trametinib. CD133-overexpressing conditionally reprogrammed MIC cells showed increased cell viability and reduced apoptosis in response to trametinib. CRISPR-Cas9 knockdown of CD133 reverted this phenotype in different patient-derived melanoma lines BAKP and POT. Further, doxycycline- inducible expression of CD133 attenuated trametinib-induced apoptosis and elevated levels of anti-apoptotic pAKT and BCL-2 family proteins. Thus, CD133 may play an essential role in chemoresistance against current targeted therapies, through an AKT-BCL-2-mediated pathway, representing an attractive target for intervention.

\section{Results}

2.1. CD133 overexpression in conditionally reprogrammed BAKP melanoma cells (BAKR) increases cell viability and inhibits apoptosis in response to trametinib or DTIC.

When patient-derived melanoma cells harboring BRAFWT/NRASQ61K mutation (BAK parental (BAKP) cell line) were magnetically sorted into CD133(+) and CD133(-) subpopulations and subcultured for 2 weeks, we noted a loss of CD133 expression in the CD133(+) cells. Conditional reprogramming of CD133(+) cells allowed sustained CD133 expression ( $\sim 85 \%$ CD133-positivity) together with increased expression of the stem cell markers Oct4, Nanog, and vimentin, markers of epithelial-mesenchyme transition (EMT), as well as hallmarks of melanoma initiating cells (MICs), such as melanosphere formation and chemoresistance to MAPK inhibitors [43]. CD133(+) cells may therefore represent cancer stem cells or MIC, exhibiting enhanced tumorigenesis, increased expression of cancer stem cell and EMT markers, melanosphere formation, and chemoresistance to MAPK inhibitors. The role of CD133 in development of chemoresistance was thus examined using this enriched population of conditionally reprogrammed BAKR melanoma cell line. Immunoblot analysis with antibodies to CD133, normalized to loading control $\beta$-Actin, verified CD133 overexpression in the BAKR compared to BAKP cells (Figure 1a). BAKP and BAKR cells were then exposed to increasing doses of trametinib or DTIC for $72 \mathrm{~h}$. XTT cell viability assays to assess drug sensitivity revealed that, compared to BAKP, comprised primarily of CD133(-) cells, CD133- overexpressing BAKR cells were more resistant to the drugs, exhibiting significantly higher cell viability after exposure to trametinib (Figure 1b) or DTIC (Figure 1c),

Effects of CD133 overexpression on trametinib-induced apoptosis was further assessed by cell cycle analysis following treatment of cells with $100 \mathrm{nM}$ trametinib for $72 \mathrm{~h}$. 
Whereas $50 \%$ of the BAKP cells had sub-G1 DNA content indicative of apoptotic cells after trametinib treatment, this sub-G1 population significant decreased to 5\% in CD133-overexpressing BAKR cells, concomitant with a five-fold increase in the proliferative S-phase population (Figure 1d). Cells were then exposed to increasing doses of trametinib for 72 $\mathrm{h}$, and subjected to Annexin-FITC/PI staining followed by FACS analysis to detect phosphatidylserine exposed on the surface of early apoptotic cells and loss of membrane integrity in late apoptotic cells. Consistent with the XTT cell viability and cell cycle assays, FACS analysis revealed significantly higher cell viability (lower left quadrant) and lower trametinib-induced apoptosis (right quadrants), indicating increased cell survival and resistance to trametinib in CD133-overexpressing BAKR cells (Figure 1 e, f).

(a)
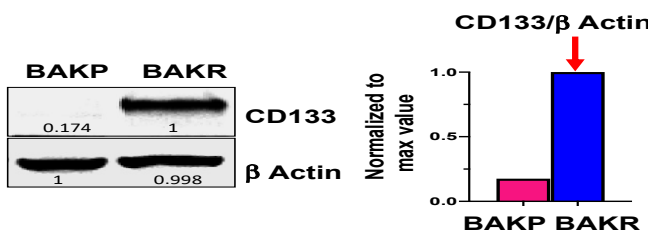

(b)

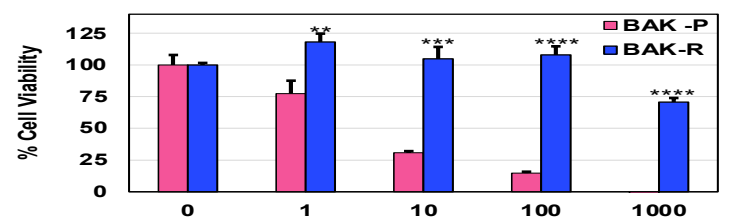

(c)

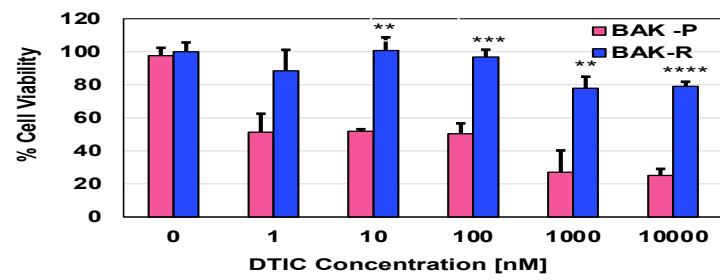

(d)

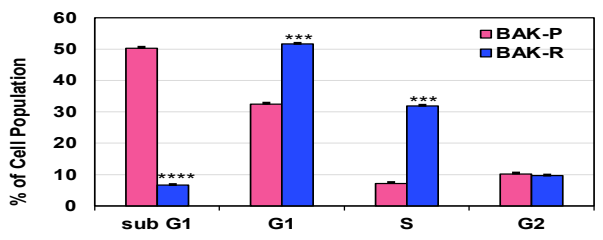

(e)

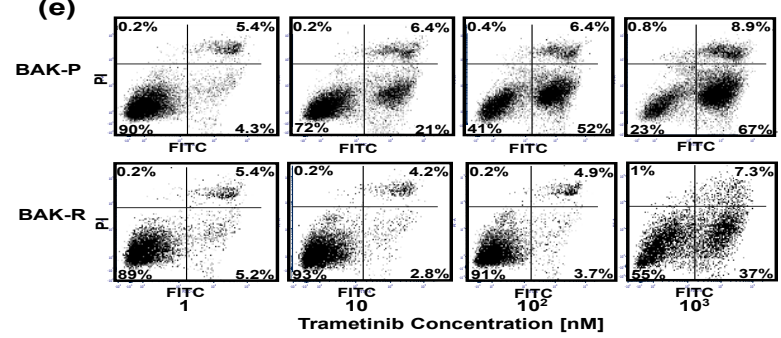

(f)

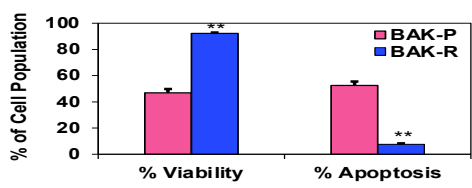

Figure 1. Sustained CD133 overexpression after magnetic sorting and conditional reprogramming in BAKR cells increases cell viability and resistance to trametinib- and DTIC-induced cell death and apoptosis. a) Immunoblot analysis show elevated CD133 expression in BAKR compared to BAKP cells. BAKR and BAKP cells were exposed to increasing doses of trametinib or DTIC for $72 \mathrm{~h}$, followed by XTT cell viability assays to assess drug sensitivity. BAKR exhibit increased resistance to trametinib (b) or DTIC (c) compared to parental cells (BAKP). (d) Cell cycle analysis of CD133(+) BAK-R melanoma cells and parental BAK-P cells after treatment with $100 \mathrm{nM}$ trametinib for $72 \mathrm{~h}$. (e) Representative dot plots of BAK$P$ and BAK-R cells treated with indicated doses of trametinib followed by Annexin-FITC/PI staining, and flow cytometry. (f) Cell Viability (\%) and Apoptosis (\%) of BAKR and BAKP cells treated with $100 \mathrm{nM}$ trametinib for $72 \mathrm{~h}$. Results shown are the means \pm SEM of three replicates of a representative experiment; essentially the same results were obtained in three independent experiments. ${ }^{* *},{ }^{* * *},{ }^{* * *}$ represent $\mathrm{p}<0.01, \mathrm{p}<0.001$, and $\mathrm{p}<0.0001$, respectively.

\subsection{CRISPR-Cas9 knockdown of CD133 in conditionally reprogrammed BAKR cells exhibit} increased proteolytic activation of apoptotic caspase-3 and caspase-mediated PARP cleavage in response to trametinib.

To address whether CD133 expression in BAKR is essential for increased survival in response to trametinib, CD133 knockdown was achieved by targeting three CD133 coding regions in exon 1 using CRISPR-Cas9 expressed in a lentiviral vector pLenti-U6-sgRNASFFV-Cas9-2A-Puro (Addgene), as previously described [43]. Four different single guide RNAs (sgRNAs; Sc, T1, T2, and T3) were used to target each exon 1 site by transduction with lentivirus expressing Cas9 together with sgRNA for the 3 targets (T1, T2, and T3) or with control SC sgRNA, followed by selection with puromycin. Due to more frameshift mutations generated in BAKR-T3 cells compared to BAKR-T1 and BAKR-T2, BAKR-T3 cells showed barely detectable CD133 protein levels, whereas BAKR-T2 cells expressed moderate of CD133 protein levels, and BAKR-T1 and BAKR-SC expressed similar CD133 
levels, comparable to those of BAK-R cells [43]. CD133-depleted BAKR-T3 cells were therefore used in subsequent experiments in this study.

RT-PCR using primers flanking the sgRNA target and immunoblot analysis verified depletion of CD133 RNA (Figure 2a) and protein (Figure 2b) in BAKR-T3 cells, compared to BAKR or scrambled sgRNA controls (BAKR-SC), after lentiviral delivery of CRISPRCas9-associated sgRNAs targeting exon 1 of the CD133 gene. CD133 knockdown in BAKR cells effectively reduced CD133 protein levels by $85 \%$, as assessed by densitometric analysis (Figure $\mathbf{2 b}$ right panel). To compare the drug sensitivities of BAKR cells expressing the gene (BAKR-SC) and CD133-depleted BAKR-T3, cells were exposed to increasing doses of trametinib and subjected to immunoblot analysis for drug-induced apoptotic cell death markers (caspase-3 proteolytic activation and PARP cleavage; Figure 2c). After proteolytic activation by initiator caspases- 8 or -9 , the executioner caspase-3 plays a central role in the caspase cascade characteristic of apoptotic pathways by cleaving various cellular protein substrates, including PARP, cytoskeletal proteins, and other executioner caspases 6 and 7. Whereas recalcitrant CD133-overexpressing BAKR-SC cells exhibited barely detectable caspase-3 activation and PARP cleavage in response to trametinib or DTIC (left panel), trametinib induced caspase-3 activation and PARP cleavage in CD133depleted BAKR-T3 cells (right panel), indicating that CD133 knockdown enhances trametinib-induced apoptosis.

To further delineate the possible mechanistic pathway by which CD133 can suppress trametinib-induced apoptosis in CD133(+) MICs, we examined the effects of CD133 depletion on levels of BCL-2 protein, an anti-apoptotic member of the BCL-2 family of proteins, that regulates apoptosis by binding to and inhibiting vital pro-apoptotic proteins BAX and BAK. Immunoblot analysis revealed a decrease in BCL-2 levels in BAKR-T3 cells, suggesting that CD133 knockdown may enhance proteolytic activation of caspase-3 and PARP cleavage in cells treated with trametinib, possibly through downregulation of BCL2. That is, CD133 may confer drug resistance to CD133(+) melanoma initiating stem cells (MICs), at least in part by increasing BCL-2 protein levels. 
(a)

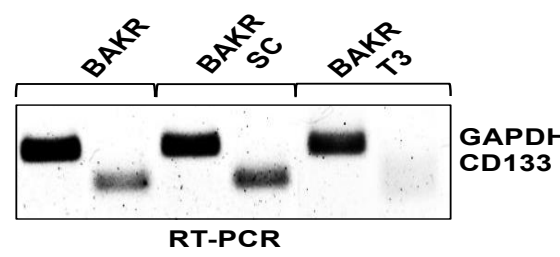

CD133/ß Actin

(b)
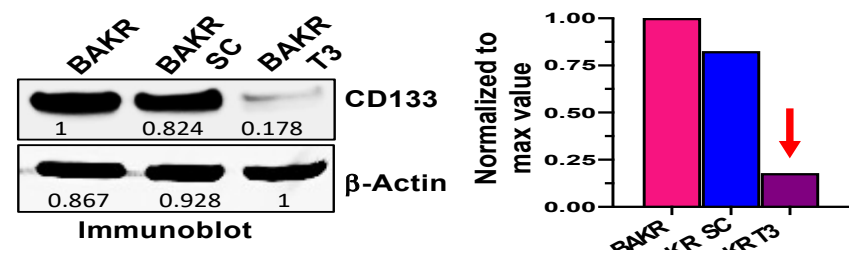

(c)

BAKR SC

BAKR T3
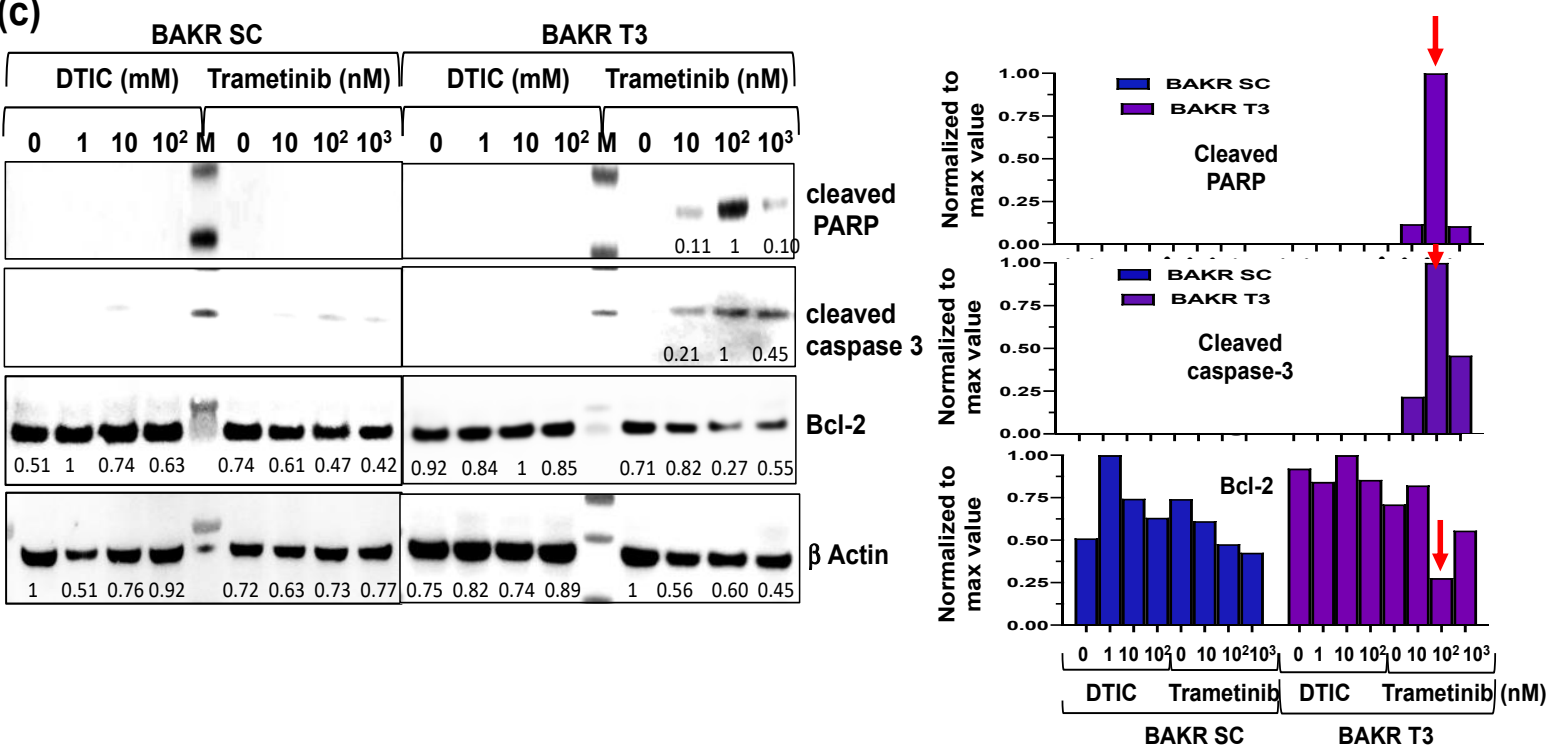

Figure 2. CRISPR-Cas9 CD133-knockdown sensitizes BAKR-T3 cells to trametinib-induced apoptosis by decreasing Bcl2 levels. CD133 expression was compared between BAKR, CRISPR-Cas9 knockdown (BAKR-T3) using specific target sgRNA, and sgRNA control (BAKR-SC) cells. RT-PCR (a) and immunoblot analysis (b) confirm depletion of CD133 RNA and protein, respectively, in BAKR-T3, compared to BAKR-SC and BAKR cells; densitometric analysis (right panel). BAKR-SC and BAKR-T3 cells were then exposed to increasing trametinib concentrations for $72 \mathrm{~h}$; cell extracts were subjected to immunoblot analysis with antibodies to the p17 subunit of the active form of caspase-3 (c). Immunoblots were stripped of antibodies and reprobed with antibodies to PARP, Bcl-2, and $\beta$-Actin for loading control. Left panels: immunoblots; right panels: densitometric analysis of immunoblots after normalization with $\beta$-actin.:.

2.3. CRISPR-Cas9 knockdown of CD133 expression in BAKP melanoma cells increases caspase 3-mediated apoptosis in response to trametinib.

CD133-associated drug resistance was next investigated in the primary melanoma cell line BAKP, harboring a BRAF ${ }^{\mathrm{WT}} / \mathrm{NRAS}^{\mathrm{Q} 61 \mathrm{~K}}$ driver mutation signature. BAKP cells are the parental cells from which the CD133-overexpressing conditionally reprogrammed BAKR cells were derived. CD133 knockdown in BAKP cells was performed by lentiviral delivery of CRISPR-Cas9 associated sgRNAs targeting three loci (T1, T2, and T3) in exon 1 of the PROM1 gene. Similar to BAKR, CD133 knockdown was effectively achieved in the BAKP-T3 cells presumably because majority of the mutations generated in these cells were frameshift mutations, whereas mutations in $\mathrm{T} 1$ and $\mathrm{T} 2$ cells were mostly in-frame mutations [43].

As with BAKR-T3, immunoblot analysis verified an $85 \%$ reduction in immunodetectable CD133 protein after CRISPR-Cas9 knockdown of CD133 expression in BAKP-T3 cells compared to control BAKP-SC (Figure 3a). BAKP-T3 cells were therefore used for subsequent experiments. In dose response experiments, Annexin-FITC/PI staining and FACS analysis (Figure $\mathbf{3 b}$ ) and fluorometric caspase-3 activity assays (Figure 3c) reveal 
significantly elevated caspase-3 mediated apoptosis and decreased cell viability in BAKPT3 after trametinib exposure, indicating that CD133 depletion sensitizes BAKP cells to trametinib, similar to the BAKR-T3 cells. As expected, BAKP cells harboring a BRAFWTNRASQ61K driver mutation signature were resistant to the BRAF inhibitor dabrafenib, since they lack the $\mathrm{BRAF}^{\mathrm{V} 600 \mathrm{E}}$ mutation targeted by this drug.

(a) BAKP
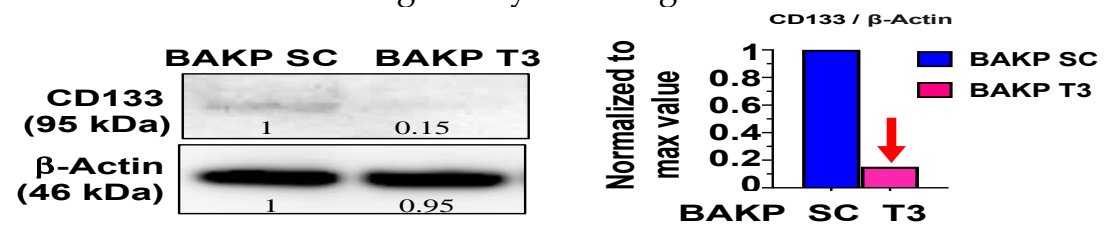

(b)
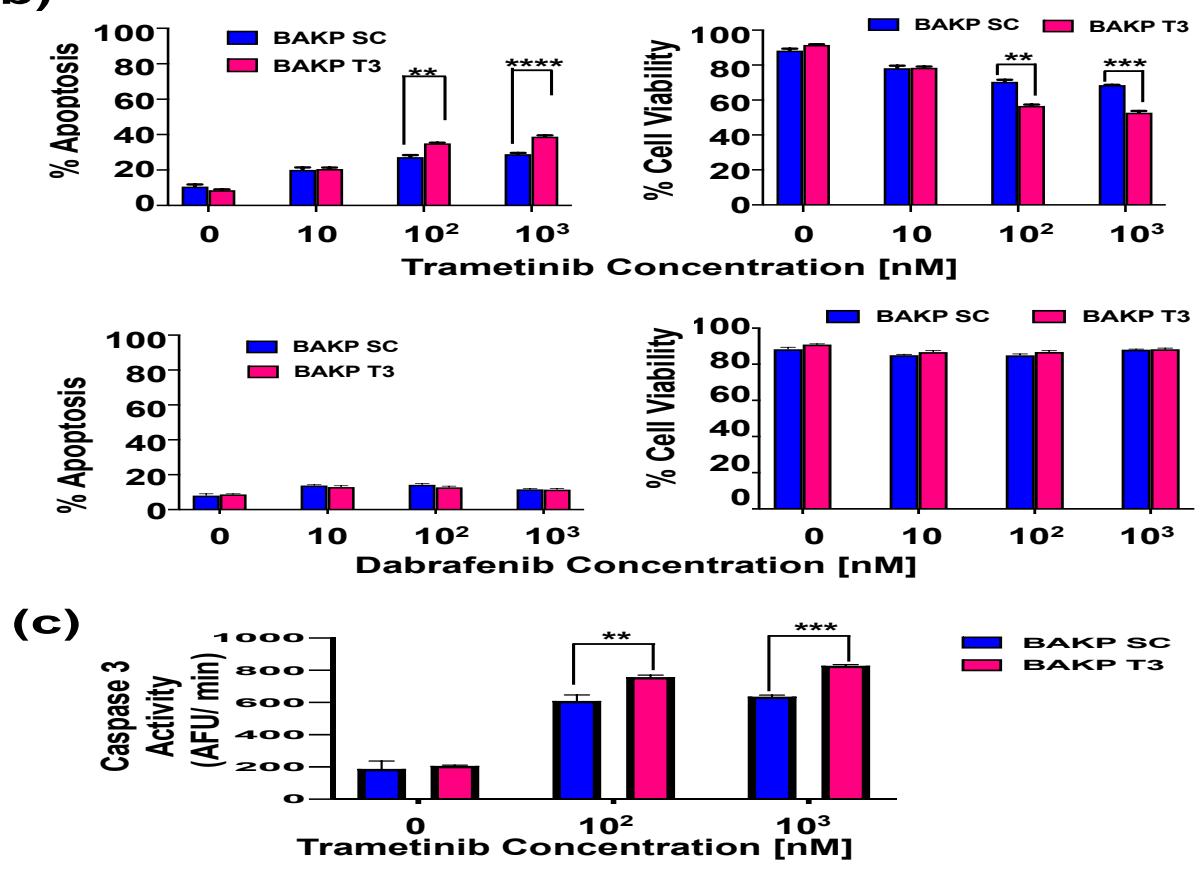

Figure 3. CRISPR-Cas9-mediated knockdown of CD133 expression in BAKP melanoma cells as confirmed by immunoblot analysis (a) increases caspase-mediated apoptosis (b, c) after exposure to trametinib. Cells treated with increasing concentrations of trametinib or dabrafenib were subjected to (b) Annexin FITC/PI apoptosis assays or (c) fluorometric caspase-3 activity assays after 72 h. CD133-depleted BAKP-T3 exhibit enhanced caspase-3 mediated apoptosis induced by trametinib, an effect that was not seen with dabrafenib. Results shown are the means \pm SEM of three replicates of a representative experiment; essentially the same results were obtained in three independent experiments. ${ }^{* *},{ }^{* * *},{ }^{* * * *}$ represent $\mathrm{p}<0.01, \mathrm{p}<0.001$, and $\mathrm{p}<0.0001$, respectively.

\subsection{CRISPR-Cas9 knockdown of CD133 expression in different melanoma cell lines BAKP and} $P O T$ reveal CD133 suppression of trametinib-induced apoptosis via modulation of BCL-2 family members

To further examine the potential mechanism(s) by which CD133 modulates the apoptotic machinery to promote cell survival in response to trametinib, CD133-depleted BAKP-T3 and control BAKP-SC cells were treated with $100 \mathrm{nM}$ trametinib for 24 and 48 $\mathrm{h}$, followed by immunoblot analysis. Consistent with the dose response experiments, BAKP-T3 exposed to $100 \mathrm{nM}$ trametinib exhibit markedly higher apoptotic PARP cleavage at $48 \mathrm{~h}$, compared to control sgRNA BAKP-SC cells (Figure 4a). POT, another drug-resistant patient-derived primary melanoma cell line that harbors an NRASQ61R mutation signature was used to validate the effects of CD133 knockdown by CRISPR-Cas9. CD133 knockdown POT cells (POT-T3) were derived and compared with POT-SC control cells. As with BAKP cells, immunodetectable CD133 decreased by 75\% in POT-T3 cells relative to POT-SC control cells (Figure $4 \mathbf{b}$ ). After incubation of CD133-depleted POT-T3 cells and POT-SC control cells with $100 \mathrm{nM}$ trametinib, immunoblot analysis revealed an early 
robust increase in apoptotic PARP cleavage by 24 hours after trametinib in CD133-depleted POT-T3 cells (Figure 4c). Sensitization to trametinib by CD133 knockdown was therefore verified in both BAKP and POT melanoma cells, suggesting that CD133 suppresses trametinib-induced apoptosis.

Interestingly, both CD133-depleted BAKP-T3 and POT-T3 cells showed elevated levels of the active form of BCL-2-associated X protein (BAX), a key pro-apoptotic member of the BCL-2 family essential for activation of the mitochondrial pathway of apoptosis (Figure 4a and 4c). Upon apoptosis induction, BAX undergoes a conformational change to its active form, translocates from the cytosol to the mitochondria, where it dimerizes with other BAX and/or BAK molecules to form oligomeric pores in the mitochondrial outer membrane, resulting in release of cytochrome $\mathrm{c}$ and other pro-apoptotic factors, leading to caspase activation. Indeed, consistent with enhanced BAX activation, a 2- to 6-fold higher proteolytic activation of caspase-3 was noted in CD133-depleted POT-T3 cells compared with control POT-SC cells prior to and in response to trametinib treatment (Figure 4d).

Whereas levels of the active form of pro-apoptotic BAX increased, CD133 knockdown in both BAKP-T3 (Figure 4e) as well as POT-T3 cells (Figure 4f) caused a 50\% reduction in expression of anti-apoptotic members of the BCL-2 family such as BCL-xL (B-cell lymphoma-extra-large) relative to control cells after trametinib treatment. An anti-apoptotic member of the BCL-2 family, BCL-xL heterodimerizes with and inhibits the activity of $\mathrm{BAX}$, thus, the increase in BAX-triggered apoptosis can at least in part be attributable to lower BCL-xL protein levels resulting from CD133 knockdown. In addition, compared with control cells, phosphorylation of the pro-survival form of BCL-2 associated agonist of cell death (BAD), phosphorylated BAD (p-BAD) was also 50\% lower in both CD133depleted BAKP-T3 (Figure 4e) and POT-T3 cells (Figure 4f) following exposure to trametinib. Dephosphorylated BAD in CD133-depleted cells can heterodimerize with and inactivate anti-apoptotic BCL-2 and BCL-xL, preventing them from binding and inhibiting $\mathrm{BAX}$, consequently, promoting BAX-initiated apoptosis.
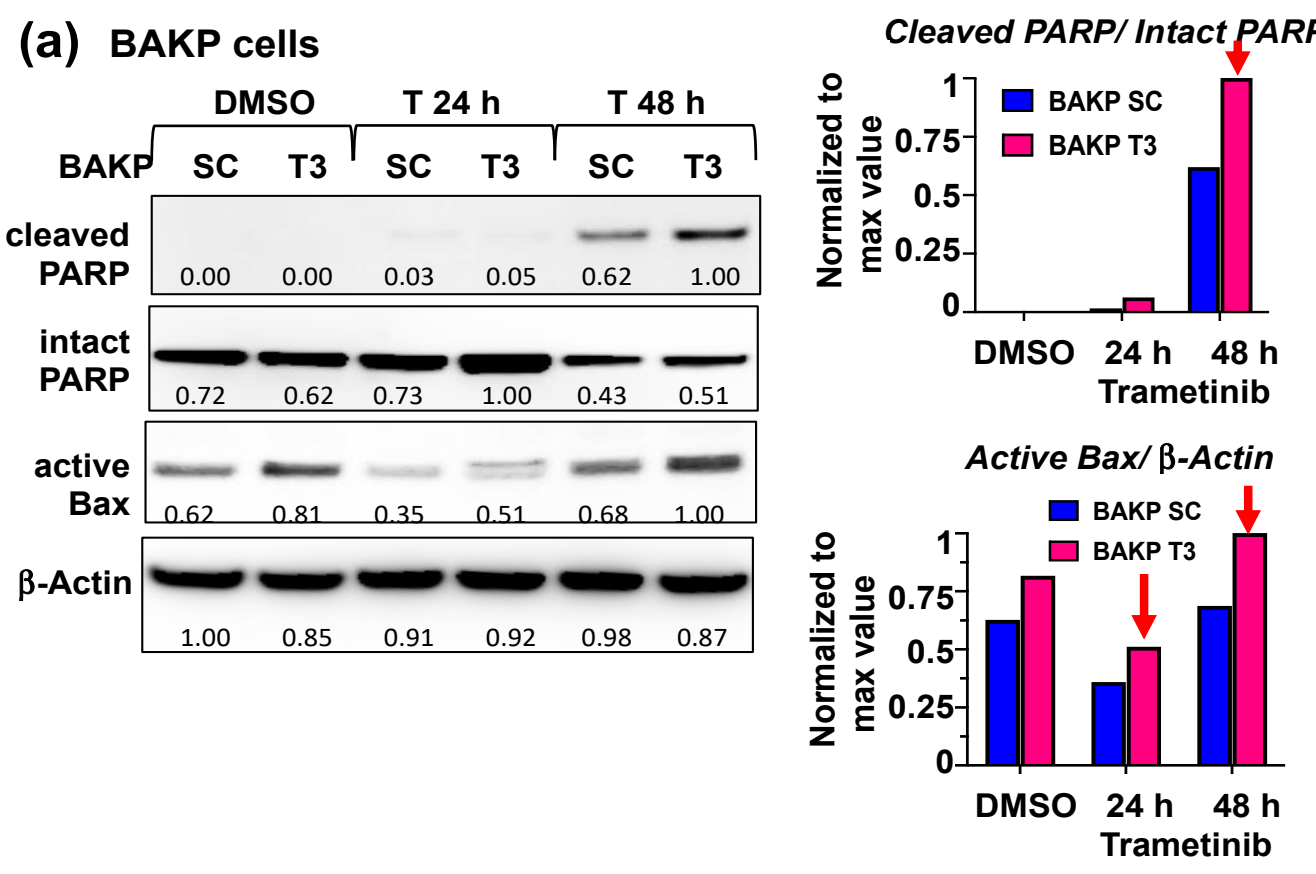
(b) POT cells
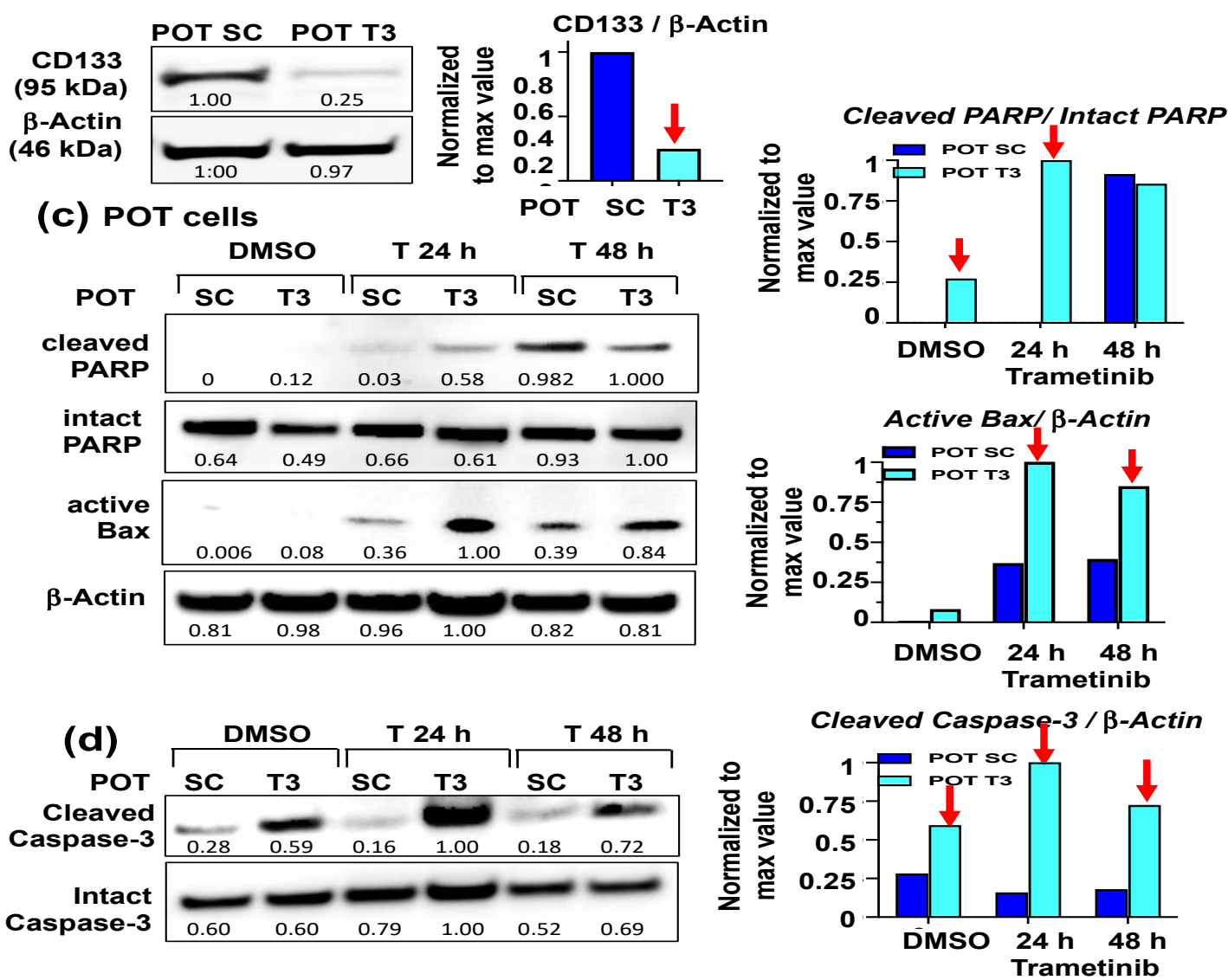

\section{(e) BAKP}
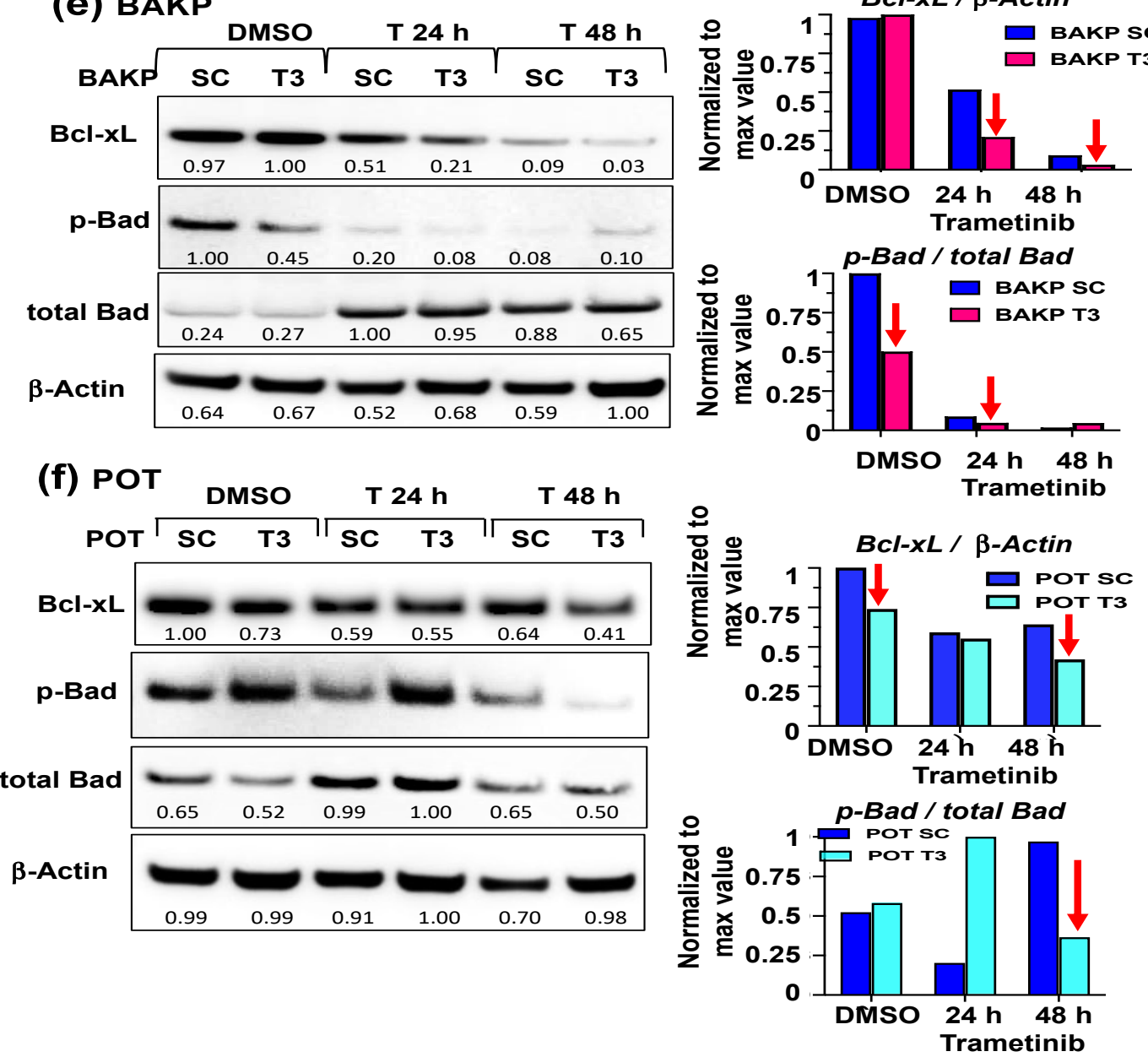
Figure 4. CRISPR-Cas9-mediated knockdown of CD133 expression in BAKP (a) and POT (c) cells markedly increases PARP cleavage, a marker of apoptosis, as well as the active form of BAX prior to and following treatment with $100 \mathrm{nM}$ trametinib. CD133 knockdown in POT-T3 cells as confirmed by immunoblot analysis (b) also induces a 2- to 6-fold increase in levels of cleaved active form of caspase-3 before and after incubation with trametinib (d). CD133 knockdown in BAKP-T3 (e) or POT-T3 cells (f) decreases levels of anti-apoptotic BCL-2 family members such as BCL-xL as well as the pro-survival form of BAD (p-BAD). Left panels: immunoblots; right panels: densitometric analysis of proteins after normalization with $\beta$-actin.

\subsection{CRISPR-Cas9 knockdown of CD133 expression in different melanoma cell lines BAKP and} POT reveal CD133 suppression of trametinib-induced apoptosis via activation of a pro-survival AKT pathway

CD133 may suppress trametinib-induced apoptosis by enhancing BAD phosphorylation, given that CD133 knockdown suppressed BAD phosphorylation by $50 \%$ in BAKPT3 (Figure 4e) and POT-T3 cells (Figure 4f) relative to control cells. Protein kinase B (AKT) phosphorylates BAD, converting BAD into a pro-survival protein (p-BAD) that is sequestered by 14-3-3 protein, leaving BCL-2 and BCL-xL free to bind BAX, thereby inhibiting BAX-mediated apoptosis. We next examined whether increased cell survival and resistance to trametinib of CD133(+) melanoma stem cells is mediated by AKT activation via upregulation of AKT expression or phosphorylation at serine 473 (S473) in these cells. Immunoblot analysis with antibodies to AKT and p-AKT (Ser473) revealed that AKT activation, as indicated by levels of phosphorylated form of AKT (p-AKT), in CD133-depleted BAKP-T3 cells were $40 \%$ lower than control BAKP-SC cells, prior to and $24 \mathrm{~h}$ after trametinib treatment (Figure 5a). Likewise, CD133-depleted POT-T3 cells exhibited a 90\% reduction in $\mathrm{p}$-AKT levels compared to control cells before or after exposure to trametinib (Figure 5b). Total AKT levels were also decreased by CD133 depletion in BAKP-T3, but not in POT-T3 cells after trametinib exposure. Diminished levels of anti-apoptotic prosurvival proteins such as BCL-xL as well as suppression of AKT activation, as indicated by reduced $\mathrm{p}-\mathrm{AKT}$, can sensitize POT as well as BAK melanoma cells to trametinib-induced apoptosis.

Previous studies have highlighted a role for CD133 in activation of AKT signaling survival pathways to promote tumorigenesis [47-49]. CD133 phosphorylation at Y828 facilitates binding of CD133 and the p85 subunit of PI3K (phosphoinositide 3-kinase), consequently phosphorylating and activating AKT [49]. AKT activation, in turn, confers chemoresistance by suppressing apoptosis and promoting cell survival [30] in part via phosphorylation and inactivation of pro-apoptotic members of the BCL-2 family such as BAD, a crucial component of the intrinsic cell death machinery [50]. Our results suggest that CD133 activates a survival signaling pathway where 1) increased phosphorylation and activation of $\mathrm{AKT}$ results in 2) phosphorylation and inactivation of the pro-apoptotic BCL-2 family member BAD, 3) decrease in the active form of pro-apoptotic BAX, 4) reduction of caspase-mediated PARP cleavage, indicating suppression of apoptosis and promotion of cell survival, ultimately potentially leading to multidrug resistance in melanomas. CD133 may play a role in the development of chemoresistance in melanoma by activating AKT and altering expression of BCL-2 family members. 


\section{(a) BAKP}
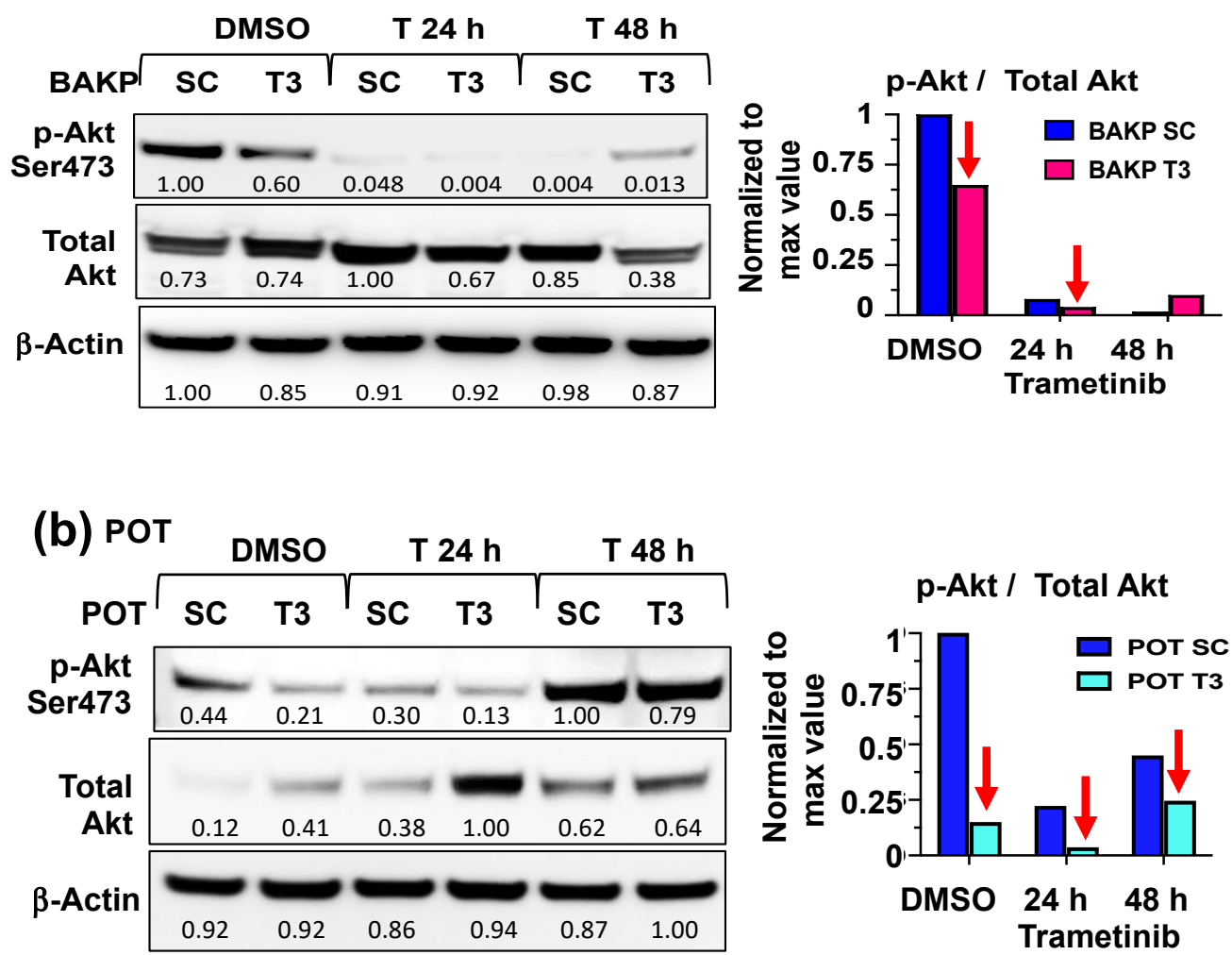

Figure 5. CRISPR-Cas9-mediated knockdown of CD133 expression in BAKP (a) and POT (b) cells markedly sensitizes cells to drug treatment by reducing anti-apoptotic phospho-AKT levels before and after exposure to $100 \mathrm{nM}$ trametinib; left panels: immunoblots; right panels: densitometric analysis of proteins after normalization with $\beta$-actin.

2.6. Dox-inducible expression of CD133 in BAKP melanoma cells suppresses trametinib-induced apoptosis, as shown by decreased caspase-3 and -9 activation and PARP cleavage, presumably via modulation of BCL-2 family members, mediated by AKT

To further confirm the mechanistic pathway between CD133, AKT activation, and the pro- and anti-apoptotic BCL-2 family proteins, BAKP and POT melanoma cells, expressing low CD133 levels, were co-transduced with a Tet activator (rtTA3) and a Tet-on vector expressing CD133 (TRE3G-CD133), or with rtTA3 alone, and selected with blasticidin and gentamycin. Stable pooled clones of BAKP-rTTA3-TRE3G-CD133 (BAKPCD133) were then incubated with or without $1 \mu \mathrm{g} / \mathrm{mL}$ doxycycline (Dox) for indicated times. Immunoblot analysis with anti-CD133 showed a marked time-dependent increase in CD133 expression by $24 \mathrm{~h}$ in the presence of Dox, with CD133 levels remaining stable for up to $72 \mathrm{~h}$ (Figure 6a). When BAKP-CD133 and BAKP- rtTA3 empty vector control cells were treated with increasing doses of trametinib $+/$ - Dox for $72 \mathrm{~h}$, as expected, BAKPCD133, but not control BAKP- rtTA3, exhibited a robust induction of CD133 expression with Dox, retaining expression even at the highest dose of trametinib tested (Figure $6 \mathbf{b}$ ). Likewise, POT- rTTA3--TRE3G-CD133 (POT-CD133) cells, a different melanoma cell line that expresses higher basal CD133 levels than BAKP cells, showed a 2-fold increase in CD133 expression in the presence of Dox (Figure 6c), confirming that CD133 expression was turned on and induced with Dox.

In dose response experiments, Dox-inducible cell lines, incubated with increasing doses of trametinib for $72 \mathrm{~h}$ with or without Dox, were subjected to Annexin-FITC apoptosis assays. While trametinib induced a dose-dependent increase in apoptosis in all cell lines, apoptotic cell death was significantly attenuated (Figure 6d), and consequently, cell 
viability increased (Figure 6e) in BAKP-CD133 and POT-CD133, but not in BAK-P-rtTA3 cells, in the presence of Dox. In addition, immunoblot analysis further shows that increased cell survival in response to trametinib corresponds with diminished levels of proteolytically-cleaved active caspase-3, cleaved PARP (Figure 7a), as well as pro-apoptotic BAX (Figure 7b) in BAKP-CD133 cells, but not in BAK-P-rtTA3 control cells incubated with Dox. In contrast, anti-apoptotic BCL-xL protein levels decreased with increasing doses of trametinib, a response that was reversed by Dox-induced CD133 expression in BAKP cells. CD133 therefore suppresses BAX- and caspase 3-mediated apoptosis, resulting in increased cell survival of BAKP and POT melanoma cells after trametinib treatment.

(a)
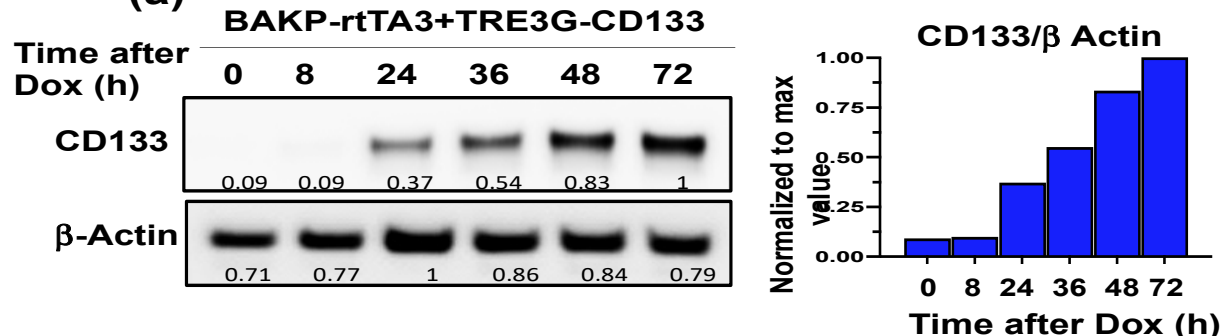

(b)

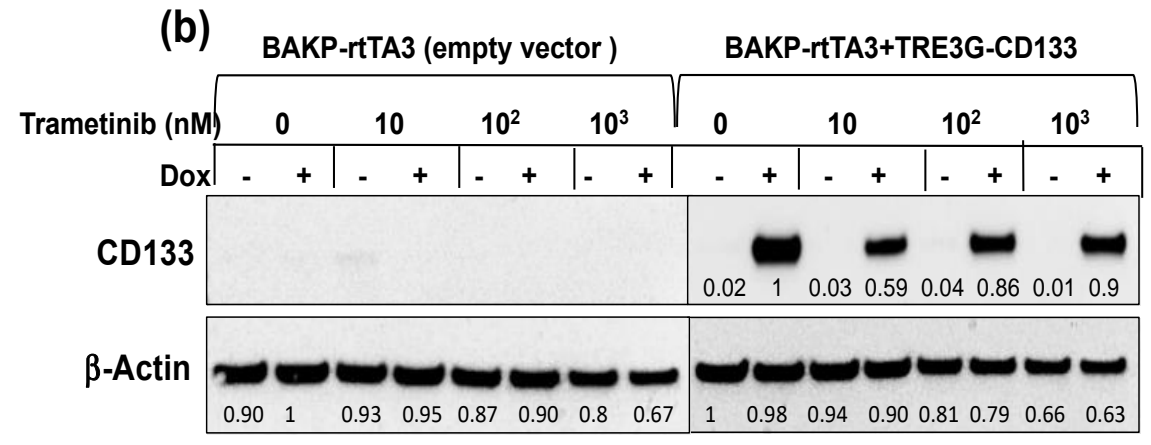

(c)
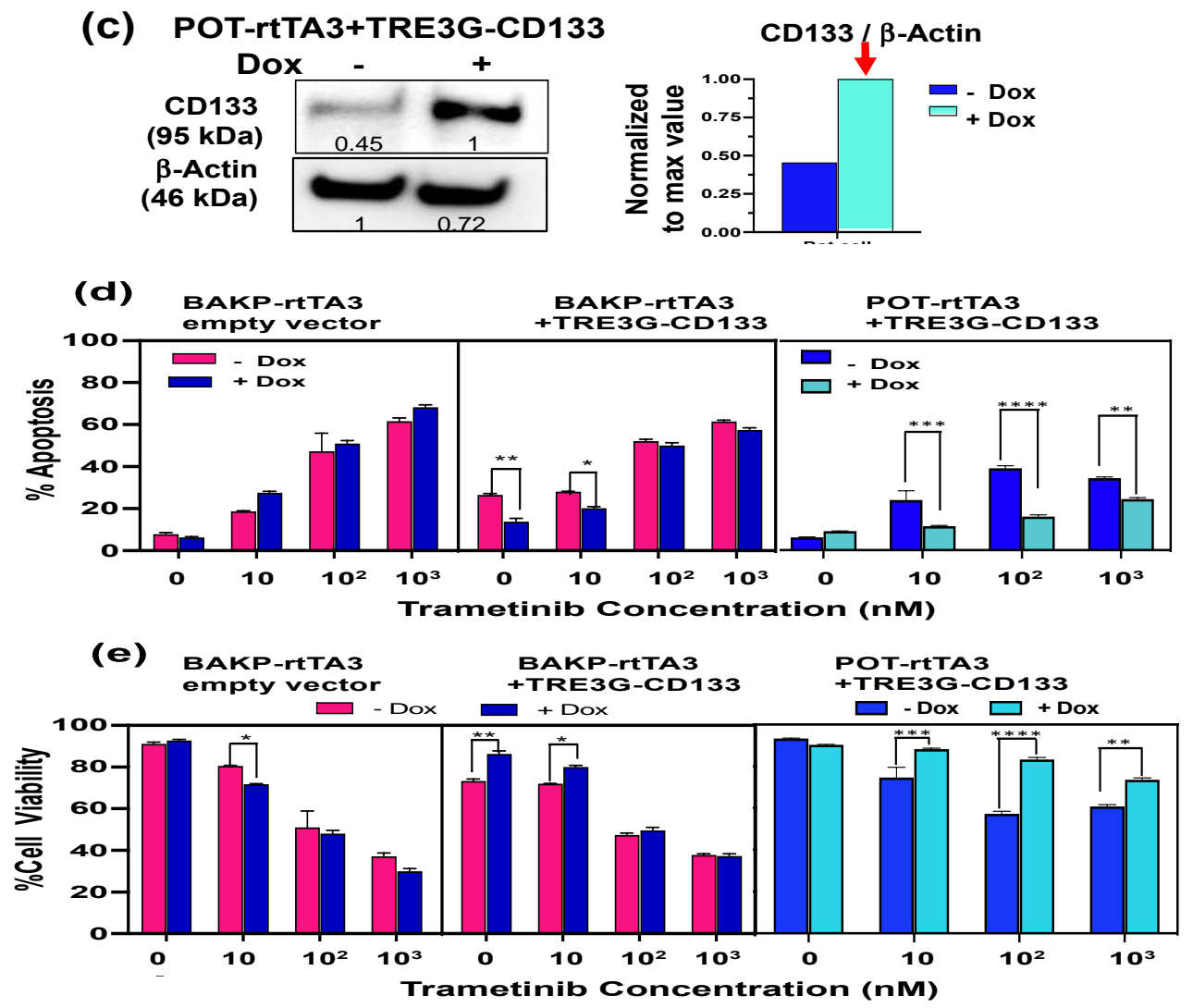
Figure 6. Dox-inducible CD133 expression in BAK-P (a, b) and POT (c) cells as verified by immunoblot analysis suppresses apoptotic cell death (d) and increases cell viability (e) in response to trametinib treatment (a) Time course of induction of CD133 expression in BAKP -CD133 cells after Dox addition. Cells were incubated with Dox for indicated time periods, and subjected to immunoblot analysis with antibodies to CD133 and $\beta$-actin. (b) BAKP CD133 as well as empty vector control BAKP-rtTA3 cells were treated with increasing concentrations of trametinib +/- Dox, followed by immunoblot analysis. (c) POT-CD133 cells were incubated $+/$ - Dox for $72 \mathrm{~h}$, and subjected to immunoblot analysis with anti-CD133. (d) BAKP-CD133, BAKP-rtTA3 empty vector control, and POT-CD133 cells were exposed to increasing doses of trametinib for $72 \mathrm{~h}$, and subjected to Annexin FITC apoptosis assays; apoptosis (\%) and cell viability (\%) of cells are shown. Results shown are the means \pm SEM of three replicates of a representative experiment; essentially the same results were obtained in three independent experiments. ${ }^{* *},{ }^{* * *},{ }^{* * * *}$ represent $\mathrm{p}<0.01, \mathrm{p}<0.001$, and $\mathrm{p}<0.0001$, respectively.
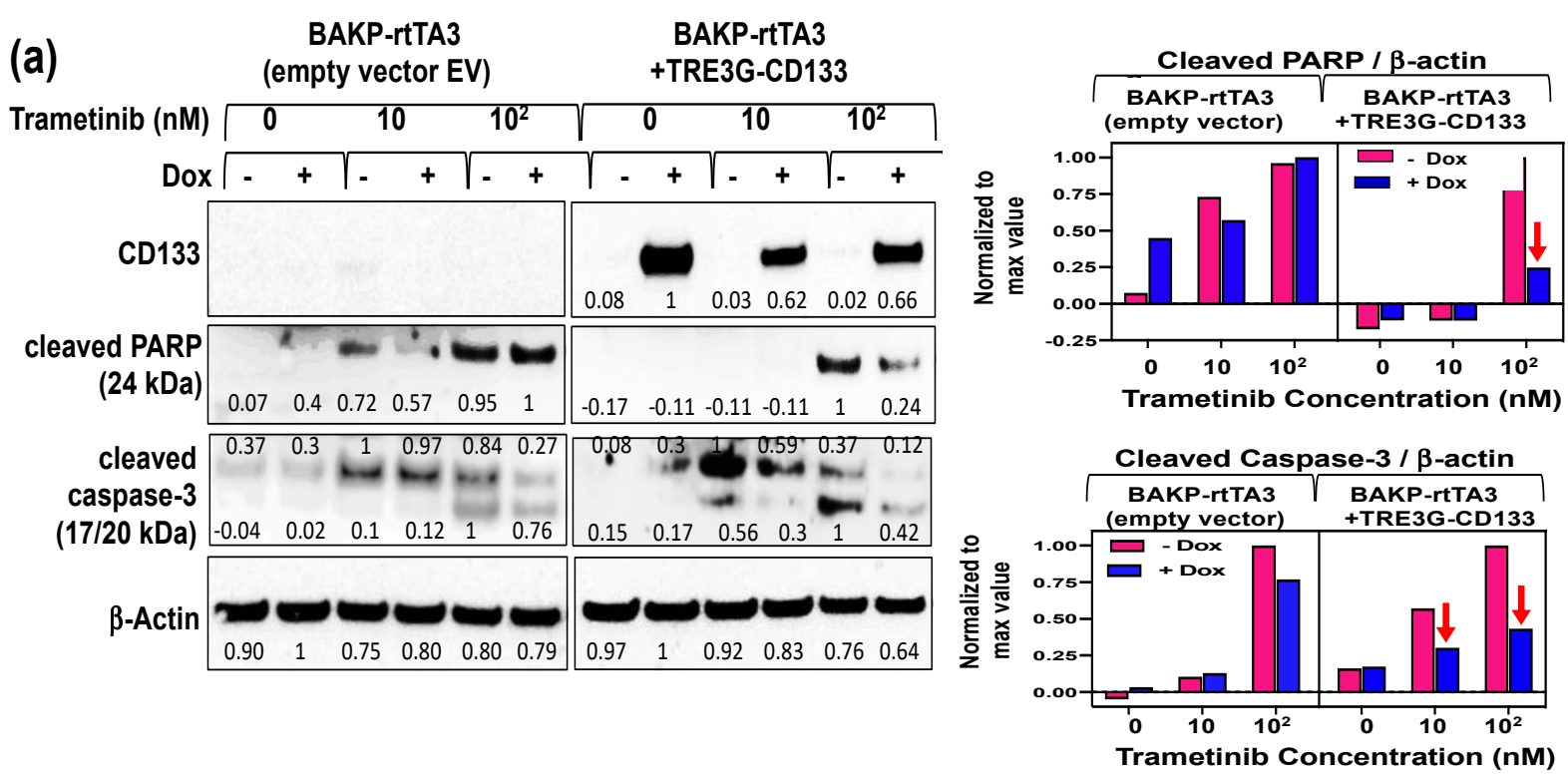

\section{(b)}

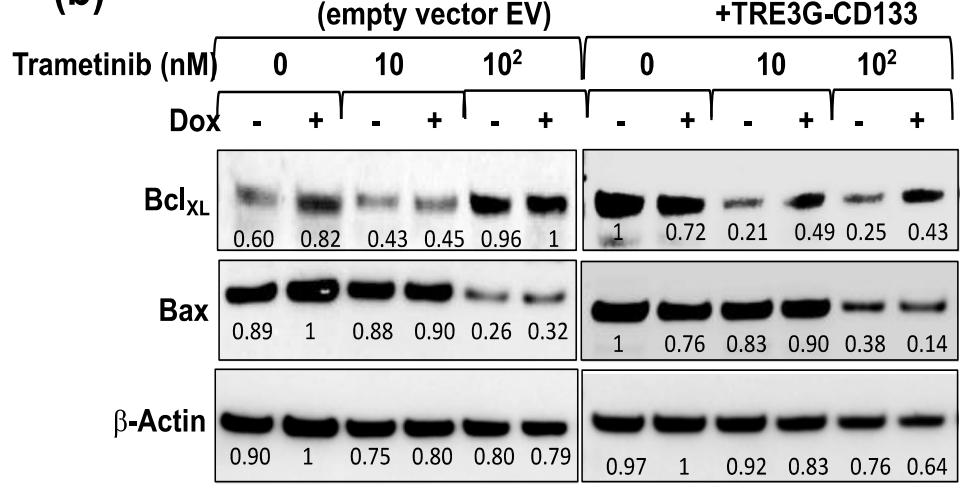

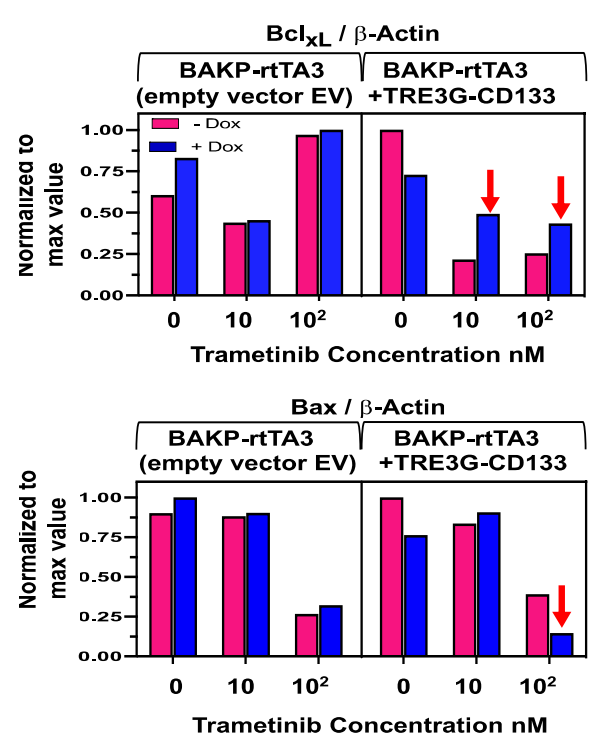

Figure 7. Dox-inducible CD133 expression in BAKP-CD133 cells suppresses (a) PARP cleavage, proteolytic activation of caspase 3 , and (b) activation of Bax, and upregulates anti-apoptotic BCL$\mathrm{xL}$ expression in response to trametinib. BAKP-CD133 as well as empty vector control BAKPrtTA3 cells were treated with increasing concentrations of trametinib $+/$ - Dox for $72 \mathrm{~h}$, followed by immunoblot analysis with antibodies to (a) cleaved PARP, cleaved caspase 3, (b) BCL-xL, active 
$\mathrm{BAX}$, and $\beta$-actin for normalization. Left panels: immunoblots; right panels: densitometric analysis of proteins after normalization with $\beta$-actin.

Dox-inducible cell lines were next used in time course experiments. Cells were treated without or with Dox to induce CD133 expression and exposed to $100 \mathrm{nM}$ trametinib for 24 or 48 hours to induce apoptosis. Immunoblot analysis at indicated time points after drug treatment assessed differences in expression levels of the apoptotic markers cleaved PARP and cleaved caspases- 3 and 9, pro-apoptotic or anti-apoptotic members of the BCL-2 family proteins BAX, BAD and p-BAD, BCL-2 and BCL-xL, as well as AKT and its activated form (p-AKT). As expected, trametinib-induced apoptosis increased timedependently, with a $90 \%$ reduction in intact PARP by $48 \mathrm{~h}$, as it was cleaved by caspase 3 to its $89 \mathrm{kDa}$ cleaved form (Figure 8a), concomitant with a robust proteolytic activation of caspase- 3 , as indicated by a $60 \%$ reduction of intact caspase- 3 and a corresponding increase in its cleaved active form by $48 \mathrm{~h}$ (Figure 8b). Caspase-9, the initiator caspase that cleaves and activates caspase- 3 in the intrinsic pathway of apoptosis, is itself cleaved and activated in response to trametinib, showing an increase in the cleaved form by $24 \mathrm{~h}$ (Figure $\mathbf{8 b}$ ). Consistent with the dose response experiments, proteolytically-cleaved active forms of caspases-3 and -9, as well as cleaved PARP were clearly diminished in Doxtreated CD133-expressing cells, exhibiting 30-50\% reduction in levels, compared to cells without Dox, further verifying suppression of trametinib-induced caspase-3 mediated apoptosis by CD133.

Activation of caspase-9 requires BAX activation, which allows release of cytochrome $\mathrm{c}$ and other pro-apoptotic molecules (e.g. SMAC/DIABLO) from the mitochondria that facilitate formation of active apoptosomes needed for caspase- 9 activation. Not surprisingly, therefore, trametinib induces a $95 \%$ increase in the levels of active BAX by $48 \mathrm{~h}$, which is reversed by Dox-induction of CD133 expression, as a $40 \%$ reduction of the active form of BAX is noted in cells incubated with Dox, compared to those without Dox (Figure $8 b$ ). Consistently, levels of anti-apoptotic/ pro-survival BCL-2 family members such as BCL-2 and BCL-xL were markedly increased by $24 \mathrm{~h}$ (Figure 8c). Similarly, phosphorylation of BAD (Figure 8c), which converts the pro-apoptotic BAD to a pro-survival p-BAD protein, as well as AKT (Figure 8d), a pro-survival kinase that is activated by phosphorylation, were also substantially enhanced in BAKP-CD133 cells 24 and $48 \mathrm{~h}$ after Dox induction of CD133 expression. These results together suggest that CD133 plays a key role in apoptosis suppression and resistance to trametinib by activating an AKT/ phosphoBAD survival pathway in human melanoma cells. 
(a) BAKP cells
BAKP-rtTA3+TRE3G-CD133
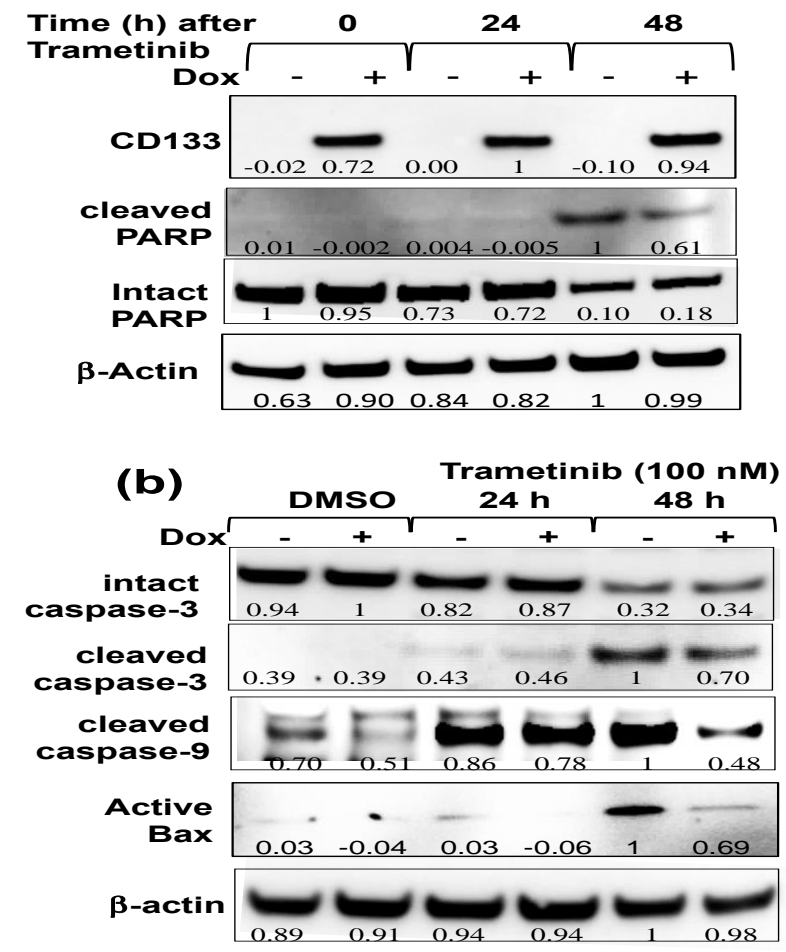
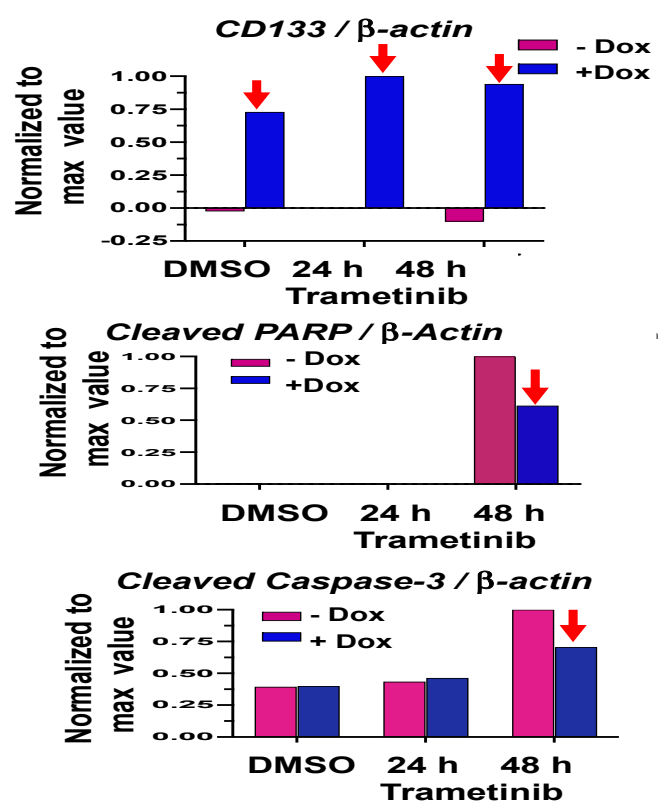

Cleaved Caspase-9/ß-actin
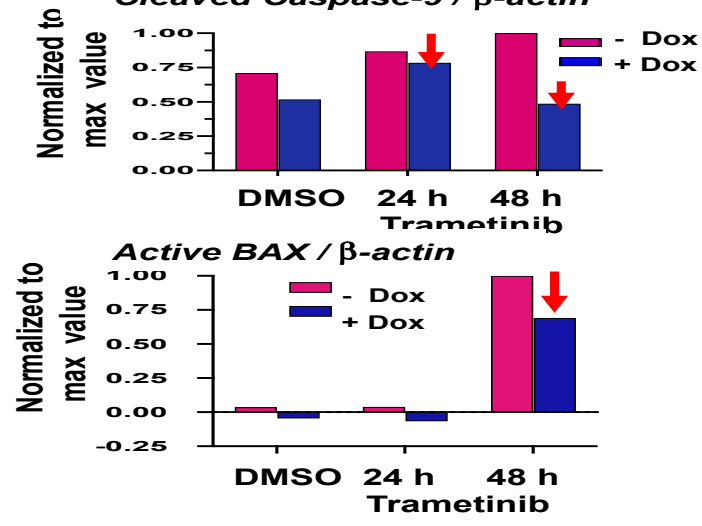

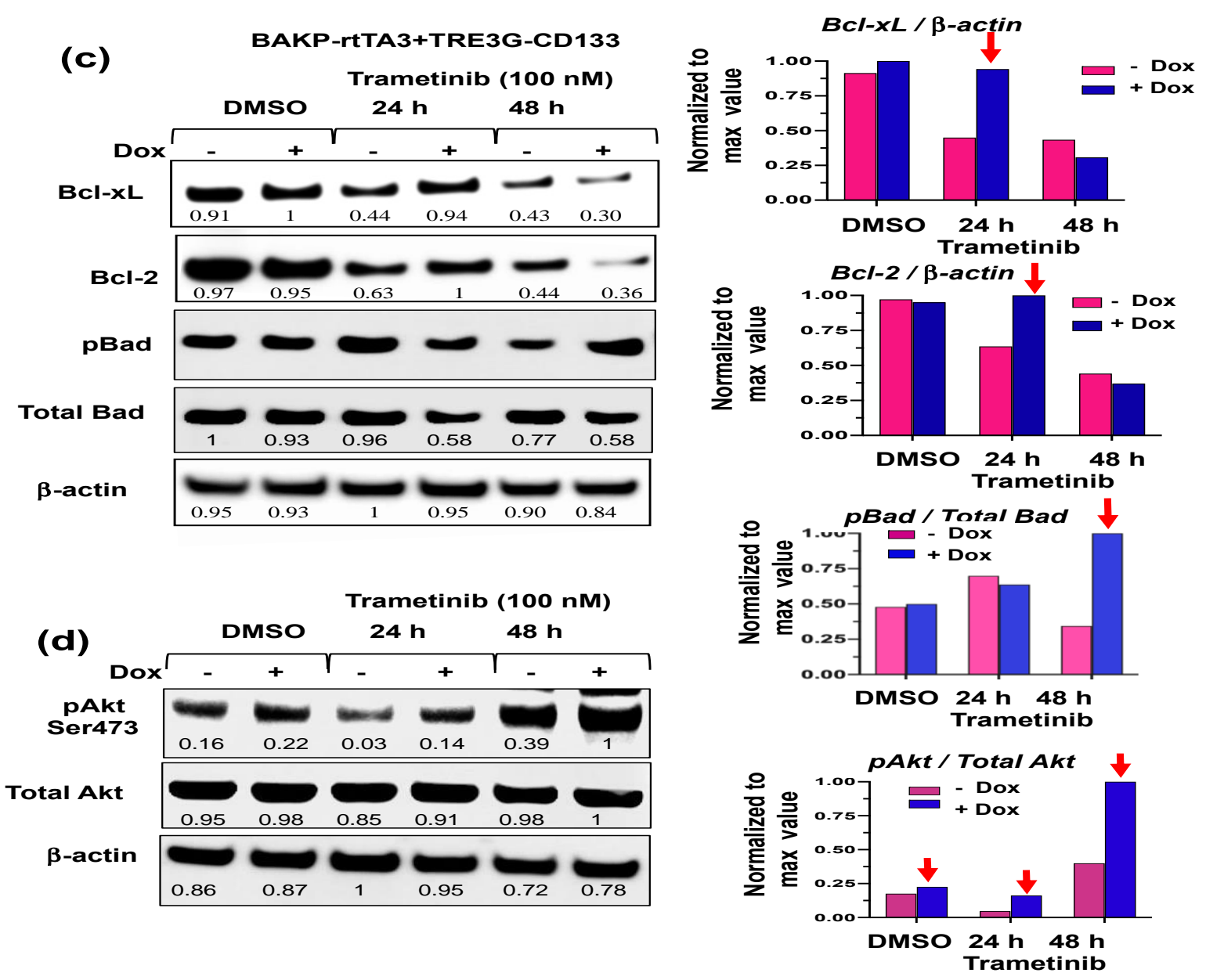

Figure 8. Dox-inducible CD133 expression attenuates trametinib-induced apoptosis in BAKPCD133 cells, as shown by (a) reduced PARP cleavage, (b) diminished proteolytic activation of caspases-3 and -9 and (c) lower levels of the active form of Bax probably mediated by upregulation of (c) anti-apoptotic proteins BCL-xL, BCL-2, p-BAD, as well as (d) p-AKT in response to trametinib treatment. BAKP-CD133 as well as BAKP-rtTA3 empty vector control cells were treated with $100 \mathrm{nM}$ trametinib for the indicated times +/- Dox, followed by immunoblot analysis with antibodies to (a) cleaved and intact PARP, (b) cleaved and intact caspase-3, cleaved caspase-9, active Bax, (c) BCL-xL, BCL-2, and BAD and p-BAD, (d) AKT and p-AKT, as well as $\beta$-actin for normalization. Left panels: immunoblots; right panels: densitometric analysis of proteins after normalization with $\beta$-actin.

2.7. Similar to BAKP cells, Dox-inducible expression of CD133 in another melanoma cell line POT suppresses trametinib-induced apoptosis via modulation of BCL-2 family members, mediated by $A K T$

To further validate the effects of increased CD133 expression on trametinib-induced apoptosis, AKT activation, levels of pro- and anti-apoptotic BCL-2 family proteins, and to reproduce the results with BAKP cells, and we compared the consequences of induced CD133 expression in another Dox-inducible melanoma cell line, POT-CD133, in a time course experiment. Similar to BAKP cells, Dox-inducible CD133 expression in POT cells suppressed trametinib-induced apoptosis, as indicated by decreased proteolytic activation of caspase-9 (Figure 9a), probably by sensitizing cells through upregulation of expression of anti-apoptotic proteins BCL-2, BCL-xL proteins, and increased phosphorylation of BAD to its pro-survival form p-BAD. In addition, increased phosphorylation and activation of AKT (Figure 9b) after trametinib treatment was also verified in POT-CD33 cells in the presence of Dox $48 \mathrm{~h}$ after trametinib treatment. Attenuation of trametinibinduced apoptosis in both Dox-treated CD133-expressing BAKP-CD3133 as well as POT- 
CD133 cells compared to cells without Dox, and the reproducible effects of CD133 expression on BCL-2 family members and AKT activation in different melanoma cell lines indicate that these responses are not cell line specific, and lend support to a role for CD133 in apoptosis suppression and increased survival after trametinib in different melanoma lines.
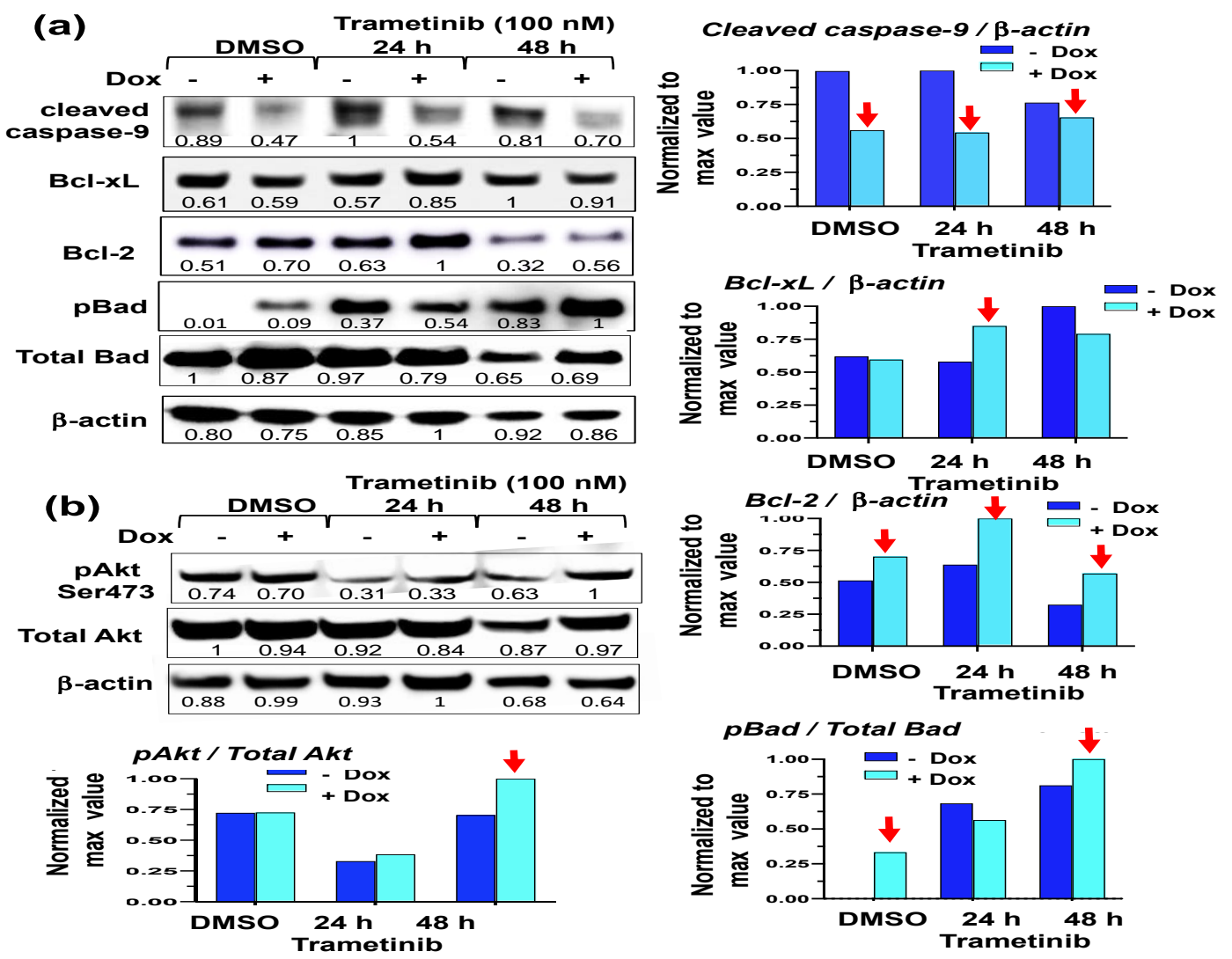

Figure 9. Dox-inducible CD133 expression attenuates trametinib-induced apoptosis in POT-CD133 cells, as shown by diminished proteolytic activation of caspase-9 (a), probably mediated by upregulation of anti-apoptotic proteins BCL-xL, BCL-2, p-BAD, BAD, as well as (b) p-AKT in response to trametinib treatment. POT-CD133 cells were treated with $100 \mathrm{nM}$ trametinib for the indicated times +/- Dox, followed by immunoblot analysis with antibodies to (a) cleaved active caspase-9, BCL-xL, BCL-2, and BAD and p-BAD, (d) AKT and p-AKT, as well as $\beta$-actin for normalization. left panels: immunoblots; right panels: densitometric analysis of proteins after normalization with $\beta$-actin.

2.8. CD133 increases resistance of melanoma cells to trametinib by blocking apoptosis in an $A K T / p-B A D$ - survival pathway, as assessed by siRNA knockdown of AKT expression and use of $B C L-2$ inhibitor ABT-263

We further examined the potential molecular mechanism(s) of CD133-associated inhibition of apoptosis, using the CRISPR-Cas9 knockdown BAKP-T3 and POT-T3 cells or their respective controls, BAKP-SC and POT-SC cells. CD133 binds to and activates phosphoinositide 3-kinase (PI3K), resulting in preferential activation of $\mathrm{AKT}$, which can then phosphorylate and inactivate BAD, a pro-apoptotic member of the BCL-2 family. We wished to determine if the suppressive effects of CD133 on apoptosis are mediated through AKT activation, followed by BAD phosphorylation, or by upregulation of antiapoptotic BCL-2 family members. Thus, we modulated AKT1/2 expression and activity by AKT siRNA knockdown (Figure 10), or inhibited anti-apoptotic BCL-2 family members with ABT-263 (Figure 11).

Immunoblot analysis confirmed successful AKT1/2 knockdown by siRNA in BAKPT3 and POT-T3 cells or their respective controls BAKP-SC and POT-SC cells (Figure 10). 
Compared to scrambled controls siRNA knockdown of AKT1/2 decreased levels of phospho-BAD, suggesting that AKT knockdown by siRNA can restore trametinib-induced apoptosis suppressed by CD133. CD133 knockdown lowers phospho-Bad levels in control or AKT siRNA knockdown cells, indicating that CD133 may be upstream of AKT signaling.
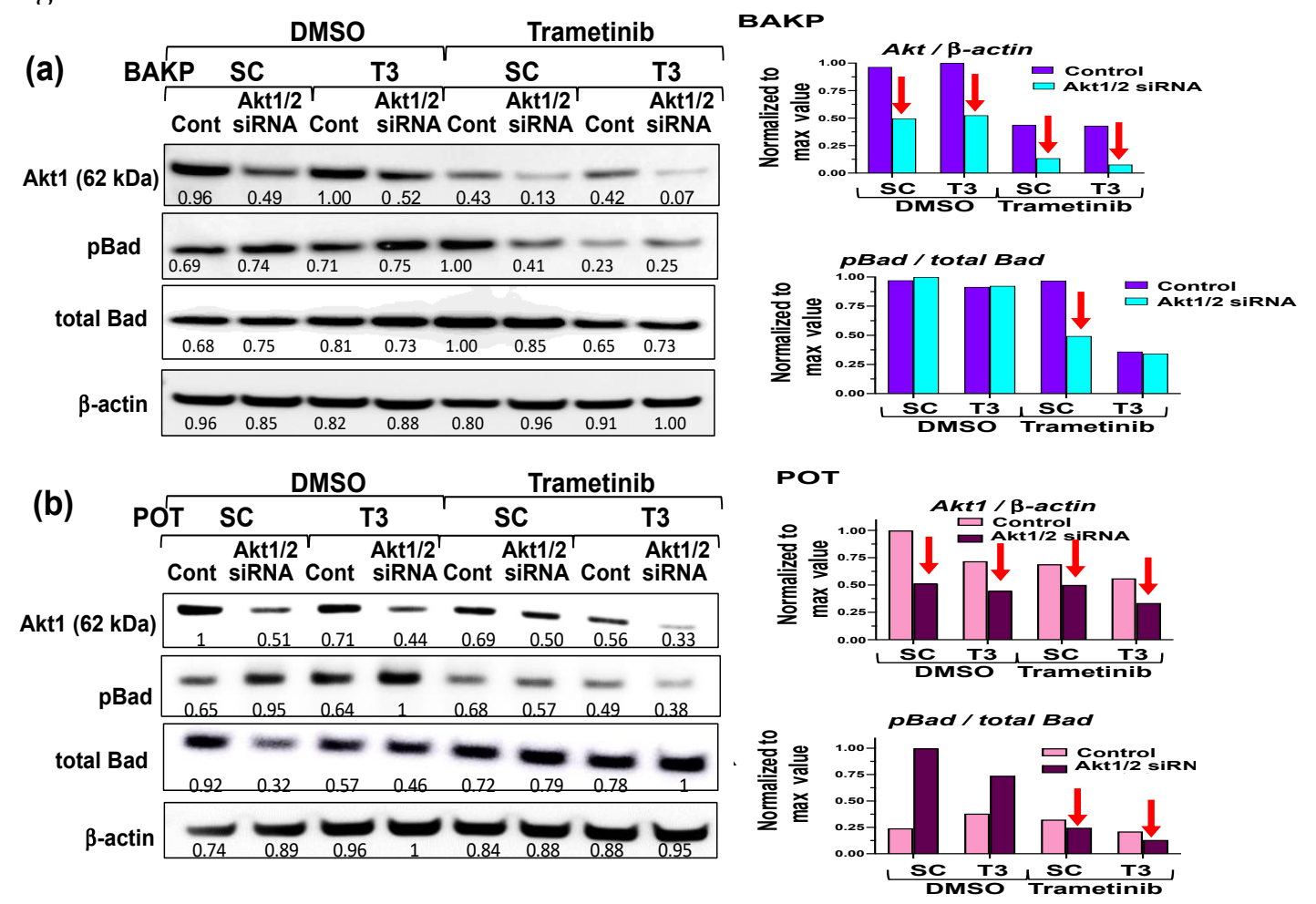

Figure 10. Immunoblot analysis of siRNA knockdown of AKT1/2 in (a) BAKP cells and (b) POT cells, reveal that AKT1/2 siRNA knockdown reduces anti-apoptotic phospho-Bad levels. BAKP-T3 and POT-T3 cells or their respective controls BAKP-SC and POT-SC cell were transfected with AKT1/2 siRNA pool or the scrambled control siRNA ("Cont"). $48 \mathrm{~h}$ after transfection, cells were treated with $100 \mathrm{mM}$ trametinib for $24 \mathrm{~h}$, and subjected to immunoblot analysis with antibodies to AKT1, BAD, p-BAD, cleaved PARP, intact PARP, or $\beta$-actin. left panels: immunoblots; right panels: densitometric analysis of proteins after normalization with $\beta$-actin.

To further investigate the interaction between CD133 and anti-apoptotic BCL-2 family members, BAKP-T3 and BAKP-SC cells were treated with $100 \mathrm{nM}$ trametinib or ABT263 alone, or in combination (Figure 11). Maximal apoptosis can only be induced by either a combination of CD133 knockdown plus MAPK inhibition by trametinib, or BCL-2 family inhibition by ABT-263 plus trametinib. CD133 knockdown in BAK-P-T3 cells increased levels of active form of BAX after BCL-2 inhibition with ABT-263. The BCL-xL, BCL-2 and $B C L-w$ inhibitor ABT-263 mirrored the effects of CD133 knockdown. That is, in the presence of ABT-263, levels of cleaved-PARP and active BAX in BAKP-SC cells increased to the same level as that exhibited by CD133-knockdown of BAKP-T3 cells. Further, expression of BCL-xL and MCL-1 was diminished in both BAKP-SC and BAKP-T3 cells treated with a combination of T+ABT-263. In conclusion, AKT1/2 knockdown or BCL-2 family inhibition induces a significant increase in trametinib-induced apoptosis of scrambled control BAKP-SC cells, comparable to that induced by CD133 knockdown in BAKP-T3 cells. These results together indicate that CD133 can increase resistance of melanoma cells to the targeted kinase inhibitor trametinib by blocking apoptosis in an AKT/p-BAD - mediated pathway. 
(a) BAKP

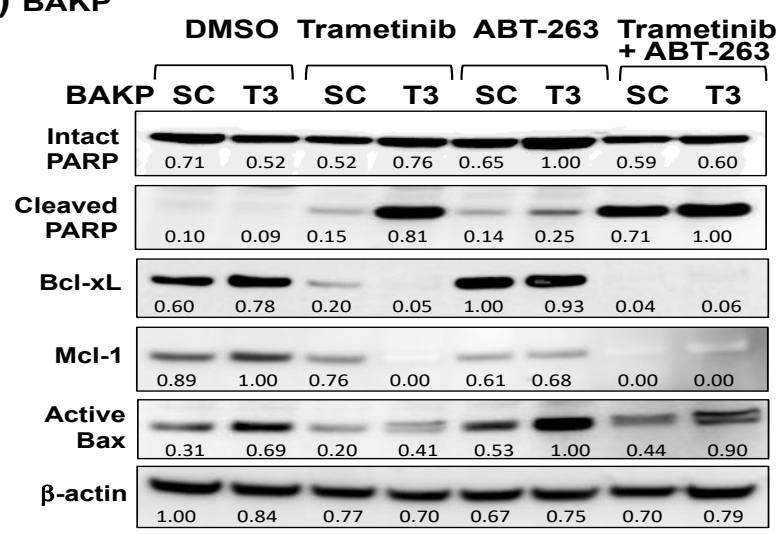

(b) POT

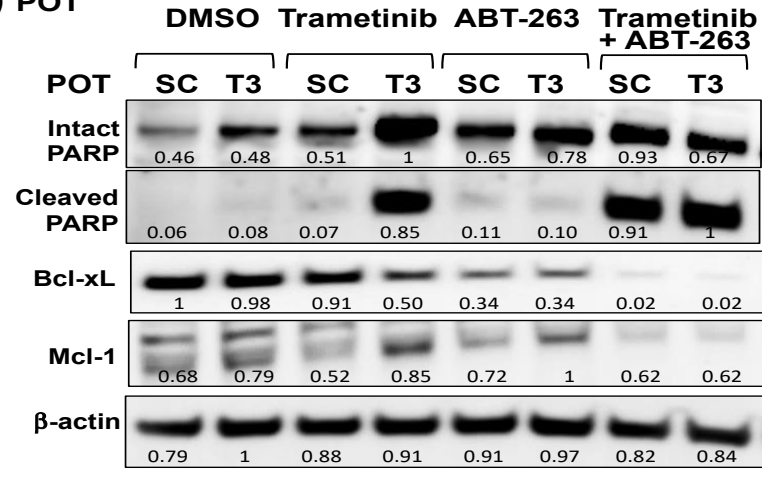

BAKP
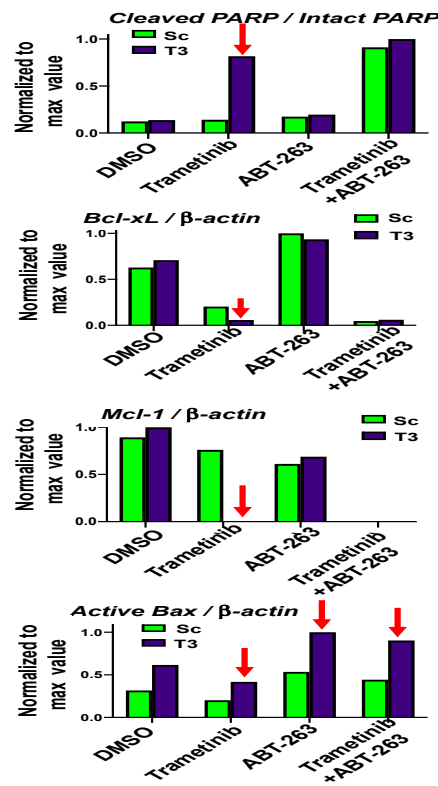

POT
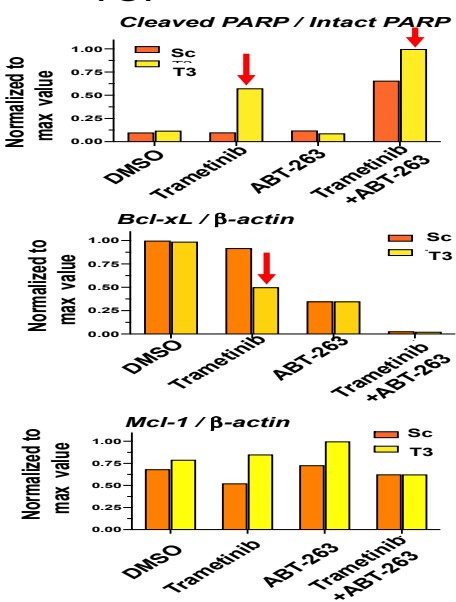

Figure 11. Immunoblot analysis of BCL-2 family inhibitor ABT-263 and/or CD133 knockdown reveal that both block the AKT pathway, inducing maximum apoptosis (PARP-cleavage) in the presence of trametinib in (a) BAKP and (b) POT cells. BAKP-T3 and POT-T3 cells or their respective controls BAKP-SC and POT-SC cell were treated with trametinib or ABT-263 at $100 \mathrm{nM}$ alone or in combination for $48 \mathrm{~h}$, and then subjected to immunoblot analysis with antibodies to cleaved PARP, intact PARP, BCL-xL, MCL-1, or $\beta$-actin. left panels: immunoblots; right panels: densitometric analysis of proteins after normalization with $\beta$-actin.

\section{Discussion}

In our previous study, we showed that CD133 levels were correlated with recurrent disease, poor clinical outcomes, and decreased overall survival; CD133(+) cells formed tumors in nude mouse xenografts, whereas CD133(-) cells did not [13]. We have also shown that expression of CD133 is associated with the upregulation of ABCBG2 and drug resistance using siRNA and pharmacological inhibitors [43]. In the current study, we utilized three different patient-derived melanoma cell lines (BAKP, BAKR, and POT) with CRISPR/Cas9-mediated CD133-knockdown, as well as Dox-inducible CD133 expression in two different cell lines (BAKP and POT), and showed that CD133 appears to play an apical role in the inhibition of an CD133/AKT/BCL-2 family-dependent pathway that works in combination with the MAPK pathway to potently inhibit active BAX/caspase-9mediated apoptosis. CD-133 knockdown induced characteristic hallmarks of apoptosis, including an increase in expression of phosphatidylserine (PS) on the outer leaflet of the 
cell membrane, proteolytic activation of caspase-3, PARP cleavage, and DNA fragmentation, the latter of which was observed in the sub-G1 population of cells (Figure 1d). Importantly, inhibition of both CD133 and MAPK pathways dramatically enhances apoptosis. For example, depletion of CD133 (e.g., BAKP-T3, POT-T3), along with inhibition of MAPK (i.e., by trametinib inhibition of MEK) resulted in a powerful apoptotic response, characterized by cleavage of the full-length $(116 \mathrm{kDa})$ PARP into $89 \mathrm{kDa}$ and $24 \mathrm{kDa}$ fragments, characteristic caspase-3/7-products as we and others have shown previously [5153]. This is strikingly apparent in Figure 11a, $\mathbf{b}$ (second panel, lanes 3 vs. 4).

DTIC did not induce apoptosis as strongly as did trametinib, although the former is a DNA alkylating agent. This may explain the failure of DTIC as the previous standard of care for melanoma, with a cure rate of only $5 \%$. While both DTIC and trametinib reduced viability, trametinib was more clearly associated with apoptosis that was regulated by CD133 (Figure 1).

We previously showed that BAKP cells maintained a population of $5 \%$ of CD133+ cells. Following MACS sorting, we were able to increase this population to $85 \%$; however, this population was unstable and the cells lost expression of CD133 within 2 weeks $[43,54]$. We therefore used conditional reprogramming to retain CD133 expression, which was stably elevated in most of these cells ([43] and Figure 1a). These BAKR cells were resistant to trametinib and DTIC, and characterized by reduced levels of apoptosis. While correlative, we knocked down CD133 in BAKR cells (BAKR-T3) and found that resistance to drug-induced apoptosis was lost. Our previous studies showed a role for $A B C$ genes. We found that additional resistance induced alterations in the levels, phosphorylation state, and balance between apoptotic proteins, which in turn were controlled by the CD133 status in the cells. Knockdown of CD133 in BAKR-T3 cells resulted in decreased phosphorylation of AKT (S473), decreased p-BAD, decreased BCL-xL levels, activation of $\mathrm{BAX}$, and increased cleavage of caspase-9, caspase-3, PARP, DNA fragmentation (sub G1), and increase phosphatidylserine on the outer leaflet of the cell membrane, as measured by the binding of Annexin V-FITC.

We next examined the BAKP and POT parental cells for the contribution of CD133 to drug-induced apoptosis. BAKP or POT cells were CD133-depleted by CRISPR-Cas9 knockdown and the two lines examined for their response to trametinib (Figures 3 and 4). CD133 knockdown in BAKP and POT cells was found to suppress phosphorylation and activation of $\mathrm{AKT}$, dephosphorylation of $\mathrm{BAD}$, diminish expression of $\mathrm{BCL}-\mathrm{XL}$, as well as upregulation and activation of $\mathrm{BAX}$, enhanced activation of caspase-3, PARP cleavage, and binding of Annexin V. Importantly, the knockdown of CD133 has a similar effect on BAKP, BAKR, and POT cells.

Since knockdown of CD133 was responsible for increased sensitivity of BAK and POT, we confirmed whether inducible expression of CD133 would render the cells more resistant to trametinib. Both cell types were confirmed to express CD133 at high levels only after induction by Dox, with a rapid time course (induction first occurred at $<24$ hours) and a relative low dose of Dox. We also determined that trametinib had no effect on the induction and further performed control experiments using control vector cells. Induction of CD133 expression in both cell types rendered them resistant to markers of apoptosis, including Annexin binding, activation of caspases-9, -3 and PARP cleavage, as well as increased phosphorylation or expression of AKT, BAD phosphorylation, and upregulation of anti-apoptotic BCL-2 family members (Figure 6). To confirm the roles of AKT and BCL-2, we used AKT siRNA or navitoclax, respectively, the latter of which is a pro-apoptotic BH3-mimetic [55] used in clinical trials for melanoma and in other cancers, along with BRAF/MEK inhibition [56]. This suggests that two pathways are active in chemoresistance of NRAS mutant melanoma, and that CD133 significantly contributes to at least one of these pathways via an AKT/BCL-2 family pathway, while the other pathway is related to the MAPK pathway. Further, these two pathways can be blocked by a combination of CD133/AKT/BCL-2 family inhibition along with suppression of the MAPK pathway. 
We showed that targeting CD133 by genetic manipulation helps to target MIC derived from different individuals. While inhibitors of CD133 have not yet been used in the clinic, there is great interest in their development in preclinical models. Most of these therapeutics currently use antibody components. Using shRNA knockdown, it was previously shown that phosphorylation of CD133 at Y828 results in association with PI3K in glioma as a mechanism for glioma stem cell renewal [49]. This is consistent with our results showing that CD133 depletion inhibits AKT phosphorylation, which in turn regulates drug resistance in melanoma via an apoptotic pathway regulated by BCL-2 family members. This is a reasonable hypothesis for what is occurring in melanoma cells that were examined in the current study. We extended these studies to melanoma using CRISPR-CAS9-mediated CD133 knockdown and Dox-inducible expression, and also examined the downstream effects on the response to drug treatment in the context of pathways leading to susceptibility to apoptosis, as well as show potential therapeutic combinations that might be considered to render the MIC population susceptible to treatment. Regarding specific therapeutics, we used drugs to inhibit the MAPK and BCL-2 family members. AKT was inhibited with siRNA, although there are a number of drugs blocking PI3K/AKT that are being tested in clinical trials for melanoma [57-59].

Regarding CD133, several therapeutics in development include de-immunized antibody derivatives such as one conjugated to cytolethal distending toxin (CDT), capable of inducing DNA damage via its nuclease activity [60], several conjugated to pseudomonas exotoxin A, inducing ADP-ribosylation of elongation factor 2 (eEF-2), one linked to nanoparticles, and several bi- and tri-specific constructs. One, anti-human $\mathrm{mAb}$ that recognizes non-glycosylated CD133 [61], was conjugated to pseudomonas endotoxin [62], and inhibited growth of two head and neck squamous cell carcinomas, without interfering with hematopoietic lineages. Chimeric Antigen Receptor (CAR)-T cells that are conjugated to or specific for CD133 have also had some initial success [48], as have NK cells targeting CD133 [60]. CD133-targeted aptamers have also been used to deliver nanoparticles to colorectal cancer cells [63], with significant growth inhibition. Our findings suggest different combinations of inhibitors that would be effective. For instance, given that T3 CD133- melanoma is sensitive to trametinib (Figures 2c, 3c, 4c), MAPK inhibitors would be promising adjuvant to these CD133-based therapies.

Future studies will attempt to connect the mechanism of upregulation with CD133 expression. Additional anti-apoptotic gene pathways will be examined using our Doxinducible BAKP-CD133 cells, including those regulated by TGF $\beta$, which we have found to be important after microarray analysis comparing BAKR vs. BAKP cells. We have also previously shown the importance of the TGF $\beta$ / inhibitor of differentiation (ID) pathway in melanomagenesis [64,65]. Given that these gene products are regulated by miRs [66, 67], the differential miR expression profiles in CD133(+) versus CD133(-) melanoma cells will be further examined.

\section{Materials and Methods}

\subsection{Cells}

Melanoma initiating cells (MIC) were obtained from patient-derived human melanoma cell lines harboring different kinase mutations including BAKP (BRAFWT, NRASQ61K) and POT (BRAFW, NRASQ61R). Cell suspensions, derived from fresh lymph node metastases from patients with short overall survival ( $<10$ months), were mechanically dissociated by mincing in Iscove's Modified Dulbecco's Medium (IMDM) supplemented with 10\% FBS and antibiotics (1\% penicillin-streptomycin). Dissociated cells were analyzed for the melanoma-specific antigens MART1 and S100 by flow cytometry using specific antibodies, and were maintained in IMDM plus $10 \%$ FBS and $1 \%$ penicillin-streptomycin, in a $37{ }^{\circ} \mathrm{C} 5 \% \mathrm{CO}_{2}$ humidified incubator. Cells were grown to $70-80 \%$ confluency and then passaged 1:4 (two population doublings). After several passages, the presence of BRAFV600 or NRASQ61 in each of the parental cell lines was verified by direct Sanger DNA sequencing of PCR products, as described previously [43]. 
4.2. Magnetic Sorting, Pre- and Post-Staining for CD133-positivity and Conditional Reprogramming of CD133(+) cells

CD133(+) and CD133(-) subpopulations were separated from early passages $(<20)$ of parental cell culture suspensions using paramagnetic nanobeads conjugated to antiCD133 and a MACS ${ }^{\circledR}$ Column (Miltenyi Biotech) according to manufacturer's protocols. Sorted cells were incubated with anti-CD133 (epitope 2) conjugated to PE (clone REA816; Miltenyi Biotec, Auburn, CA) and imaged by fluorescence microscopy to determine CD133 positivity after magnetic-activated cell sorting $\left(\mathrm{MACS}^{\circledR}\right)$. Flow cytometry was performed to confirm the sorted populations as CD133(+) or CD133(-) using mouse mAb specific for epitope 1 of CD133 (Miltenyi Biotech). To derive a subpopulation of enriched CD133(+) MIC cells with sustained CD133 overexpression, magnetically sorted CD133(+) cells were conditionally reprogrammed using the "Georgetown Method" as previously described [43]. CD133(+) 3D melanospheres were cultured for a month in 3D conditions in hypoxic $\left(2 \% \mathrm{O}_{2}\right)$ conditions at $37{ }^{\circ} \mathrm{C}$ and $5 \% \mathrm{CO}_{2}$. in ultra-low attachment plates with DMEM-F12 stem cell media, supplemented with EGF and FGF (10 ng/mL), heparin (2.5 $\mu \mathrm{g} / \mathrm{mL}), \mathrm{B}-27, \mathrm{Y}-27632(5 \mu \mathrm{M})$, and penicillin/streptomycin. Cells were then moved to attached 2D conditions with DMEM-F12/FY media, where the cells were allowed to recover and expand at $37^{\circ} \mathrm{C}, 5 \% \mathrm{CO} 2$ for a month before CD133 immunofluorescence staining.

\subsection{AKT Knockdown by siRNA}

Knockdown experiments were performed according to standard protocols using small interfering RNAs (siRNAs) specific for AKT or scrambled siRNA controls (Santa Cruz Biotech). Successful AKT knockdown was verified by immunoblot analysis.

\subsection{CRISPR-Cas9 deletion of CD133}

Three different sgRNA sequences specific for the first exon of CD133 in lentiviral vector pLenti-U6-sgRNA-SFFV-Cas9-2A-Puro (Addgene) were packaged and transduced into melanoma cells. HEK 293 packaging cells were transfected with pLenti-U6-sgRNASFFV-Cas9-2A-Puro plasmid $(4 \mu \mathrm{g})$ containing individual signal-guide sequences (sgRNA) beginning at either 8, 69, or $205 \mathrm{bp}$ downstream of the beginning of the CD133 coding sequence, all within the first exon coding sequence of CD133, as shown below: low)

sgRNA1: CAACAGGGAGCCGAGTACGA (complement of Target 1 underlined be-

sgRNA2: TTCATCCACAGATGCTCCTA (complement of Target 2 underlined below)

sgRNA3: TTACCTTCTGGGAAATCACGC (complement of Target 3 underlined below)

CD133 coding sequence of first exon with targets T1, T2, and T3 underlined:

ATG GCC CTC GTA CTC GGC TCC CTG TTG CTG CTG GGG CTG TGC GGG AAC T1

TCC TTT TCA GGA GGG CAG CCT TCA TCC ACA GAT GCT CCT AAG GCT TGG T2

AAT TAT GAA TTG CCT GCA ACA AAT TAT GAG ACC CAA GAC TCC CAT AAA GCT GGA CCC ATT GGC ATT CTC TTT GAA CTA GTG CAT ATC TTT CTT ATG TGG TAC AGC CGC GTG ATT TCC CAG AAG GTA A

T3

Lentivirus packaging plasmids were co-transfected into HEK 293 cells with using Lipofectamine LTX (Thermo Fisher Scientific), and lentivirus was harvested in media after $48 \mathrm{~h}$ and used to transduce melanoma cells as described previously [43]. Transduced cells were then selected with puromycin $(4 \mu \mathrm{g} / \mathrm{mL})$ for 5 days, and pooled and individual clones were derived and subjected to PCR analysis of genomic sequences. Alterations of the first exon were performed by PCR amplification followed by Sanger sequencing, and NGS sequencing to examine allelic or frameshift mutations (\%) at CRISPR target sites T1, 
T2, and T3. To directly observe CRISPR-Cas9 deletions in pooled cells, the following primers flanking human genomic Targets 1, 2, or 3 (T1, T2, T3) were amplified as small PCR products, where small NHEJ deletions could be visualized on $15 \%$ urea polyacrylamide gels.

Target 1-forward (F1a) TTCCCCAAGGCTTCCAGAAG
Target 1-reverse (R1a) GCCCTCCTGAAAAGGAGTTC
Target 2-forward (F2a) GAACTCCTTTTCAGGAGGGC
Target 2-reverse (R2a) GAGAATGCCAATGGGTCCAG
Target 3-forward (F3a) CTGGACCCATTGGCATTCTC
Target 3-reverse (R3a) CATTCT1TCCCTGCCATCAGC

\subsection{Generation of Dox-Inducible Cells}

Dox-inducible lentivirus that can induce CD133 expression was generated by cotransfecting pLenti-CMV-rtTA3 Blast (Addgene) and psPAX2 and pMD2.G (5 $\mu$ g each; Addgene), with or without pLV-EGFP/Neo- TRE3G-CD133 (10 $\mu$ g; VectorBuilder Inc.) into HEK293FT packaging cells using Lipofectamine LTX (Thermo Fisher Scientific) according to the manufacturer's specifications. Medium was replaced after $16 \mathrm{~h}$, and cells were incubated for another $48 \mathrm{~h}$ to generate the lentivirus. Viral supernatant was then collected, concentrated by centrifugation, and filter-sterilized prior to use. For the virus transduction, viral supernatants were added to the media (MOI=1) in 6-well plates seeded with cells. Media was replaced with cell culture media after $24 \mathrm{~h}$, and transduced cells were then selected for 10 days with blasticidin $(40 \mu \mathrm{g} / \mathrm{ml})$ and gentamicin $(1000 \mu \mathrm{g} / \mathrm{ml})$ for 10 days as previously described [43].

\subsection{Drug Treatment and Cell Viability Assays}

Cells were plated at 5,000 cells / well in 96-well plates, and triplicate wells exposed to indicated concentrations of kinase inhibitors trametinib or dabrafenib, or DTIC for up to $72 \mathrm{~h}$. Drugs were diluted and dissolved in the same final volume of DMSO, with a final concentration of $0.2 \%$ DMSO in culture medium. As negative control, cells were exposed to $0.2 \%$ DMSO alone in medium. To determine the effects of drugs on cell viability, a colorimetric XTT assay was used (Biotium, Inc.) that measures metabolic reduction of XTT by viable cells. Absorbance readings were taken every hour for up to $3 \mathrm{~h}$ after XTT reagent addition using a Victor plate reader set at $450 \mathrm{~nm}$ to obtain a slope of $\mathrm{A}_{450} / \mathrm{min}$. Each plate included wells with the drug-treated cells in triplicate, together with 6 replicates each of increasing number of cells in IMDM medium/0.2\% DMSO to generate a standard curve of A450/min vs. cell number. The standard curve was then used to generate the drug doseresponse curves. Data presented in each figure represent the mean \pm SD of each set of triplicate drug-treated cells from a representative experiment.

\subsection{Annexin V/PI staining and flow cytometry}

As a standard apoptosis assay, attached and floating cells were collected and pooled at indicated times after drug exposure, and stained with Annexin V-FITC for detection of cell surface phosphatidylserine in apoptotic cells and propidium iodide (PI) for necrotic cells (BD Biosciences, San Jose, CA), as previously described [68], followed by flow cytometric analysis in a Becton-Dickinson FACStar Plus flow cytometer.

\subsection{Fluorometric caspase-3 activity}

Cytosolic extracts were derived from pooled attached and floating cells and subjected to fluorometric caspase-3 activity assays with a fluorescent tetrapeptide substrate specific for caspases-3 (Ac-DEVD-aminomethylcoumarin (AMC, Enzo Life Sciences, Farmingdale, NY) as previously described [69]. Free AMC from cleavage of the aspartate-AMC bond was measured in a kinetic assay over $30 \mathrm{~min}$ in a Wallac Victor3 fluorometer (PerkinElmer, Waltham, MA) with emission and excitation and at 460 and $360 \mathrm{~nm}$, respectively. 
Caspase activity was determined after linear regression analysis of the initial velocity (slope) for each curve showing emission from each sample plotted against time.

\subsection{Immunoblot Analysis}

SDS-Page electrophoresis and transfer of proteins to nitrocellulose membranes were performed according to standard procedures. Ponceau S (0.1\%) staining of membranes verified transfer of proteins and equal loading. Membranes were incubated with antibodies to CD133 (Miltenyi Biotec, Auburn, CA), cleaved and intact PARP, BAD, p-BAD, BCL-XL, MCL-1 (BioLegend, San Diego, CA) cleaved active caspase-3, cleaved active caspase-9, BCL-2, active BAX (Novus Biologicals, Centennial, CO), AKT and p-AKT (Santa Cruz Biotech, Dallas, TX), or to $\beta$-Actin (ProteinTech, Rosemont, IL) as loading control. Immunoblots were sequentially reprobed with other antibodies after stripping them of antibodies. Immune complexes were detected by incubation with horseradish peroxidase-conjugated antibodies to mouse or rabbit IgG (1:3000), followed by enhanced chemiluminescence (ECL; Pierce, Rockford, IL) and imaging in a GE Healthcare Amersham Imager 600.

\subsection{Statistical Analysis}

All experiments were performed in triplicate. Error bars are reported as SEM of triplicates and $p$-values were calculated using a standardized t-test. $p$ values of $<0.05$ were considered statistically significant and represented with ${ }^{*}$, while $\mathrm{p}<.01, \mathrm{p}<.001$, or $\mathrm{p}<.0001$ are shown as ${ }^{* *},{ }^{* * *}$, or ${ }^{* * *}$ asterisks respectively. The results are representative of 3 independent experiments with reproducible results.

\section{Conclusions}

We recently showed that CD133(+) "melanoma-initiating stem cells" (MICs) are associated with tumorigenesis, metastasis, and chemoresistance, contributing to poor patient outcome. MICs express abundant CD133, form huge melanospheres, express stem cell markers, and are more invasive and resistant to kinase inhibitors. Our current study investigates the mechanistic pathway(s) by which CD133 promotes cell survival and resistance to targeted kinase inhibitors such as trametinib in melanoma. CD133-overexpressing cancer stem cells promote cell survival by suppressing trametinib-induced caspase-3 mediated apoptosis, which is reversed by CRISPR-Cas9 knockdown of CD133 expression in these melanoma cells. These results are reproduced in another patient-derived melanoma cell line. Interestingly, apoptosis suppression is regained in these cell lines by Dox-inducible expression of CD133. CD133 may increase resistance of melanoma cells to the trametinib by blocking apoptosis in an AKT/p-Bad - mediated pathway. CD133 may therefore activate a pro-survival CD133/AKT/BCL2 family-dependent pathway that works in combination with the MAPK pathway to potently inhibit active BAX/caspase-9-mediated apoptosis (Figure 12). These data strongly indicate a mechanism for CD133(+) cancer stem cells to develop resistance to trametinib in melanoma cells, and lends strong support for the use of CD133 as a target for therapeutic intervention. 


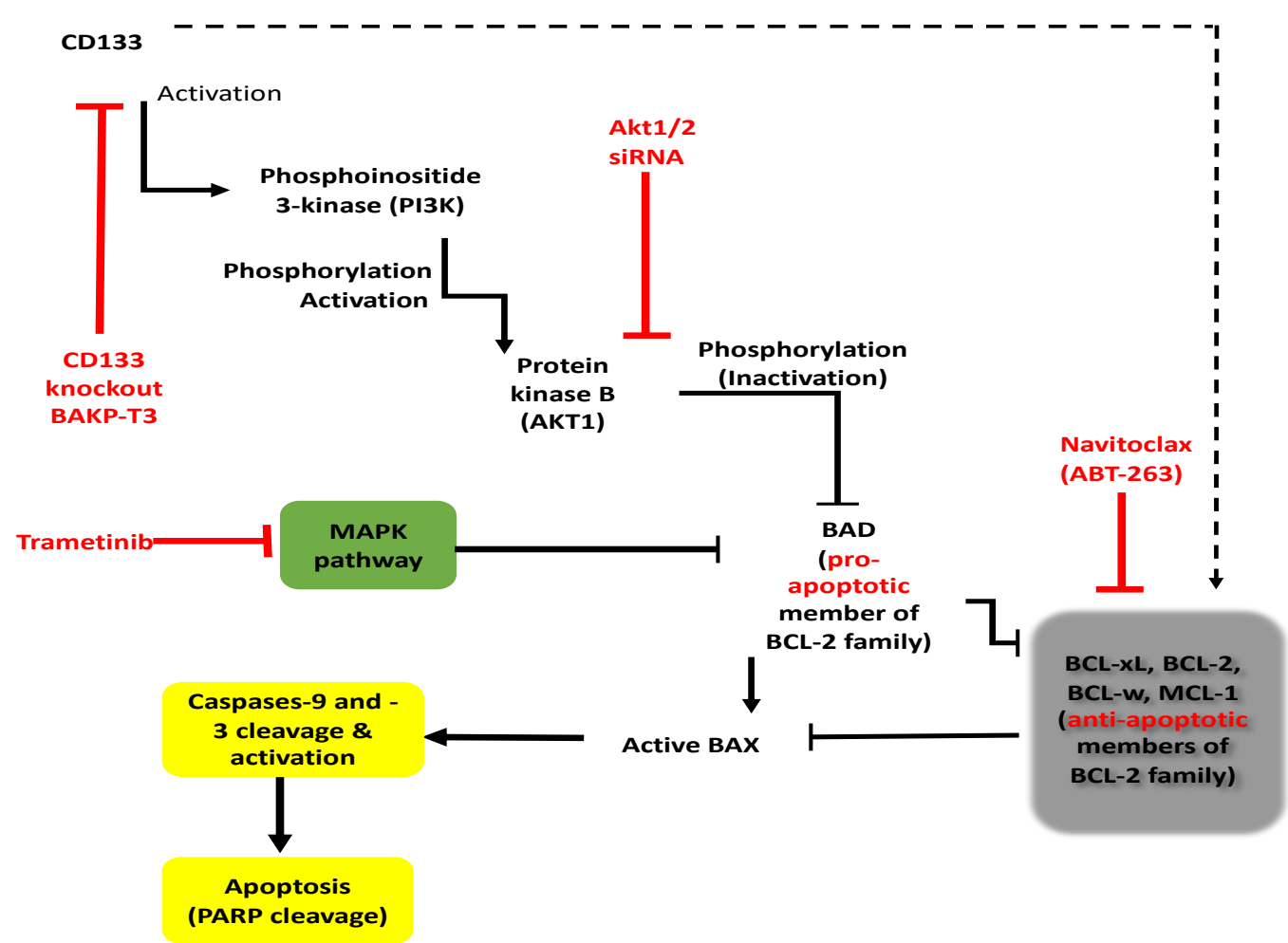

Figure 12. PI3K/Akt/ Bcl2 family pro-survival signaling pathway in CD133-positive melanoma initiating stem cells (MICs). CD133 may activate a survival pathway where 1) increased phosphorylation of AKT induces 2) phosphorylation and inactivation of BAD, 3) decrease in the active form of BAX, and 4) reduction in caspase-mediated PARP cleavage, indicating apoptosis suppression leading to drug resistance in melanomas.

Patents: None.

Supplementary Materials: S1: Scans of whole gel western blots showing molecular weight sizes of relevant proteins (indicated in arrows)

Author Contributions: conceptualization, C.S-R. and D.R..; methodology, D.R., C.S-R, Y.H., S.V.; validation, R.D., S.V., L.K., Y.H., R.A.; formal analysis, S.V., Y.H.; investigation, R.D., S.V., L.K., H.C., R.A.,Y.H.; resources, D.R., B.C., P.S., and C.S-R.; writing-original draft preparation, C.S-R. and D.R.; writing - review and editing, C.S-R., Y.H., S.V., L.K., P.S., D.R. ; supervision, D.R., C.S-R, S.V., Y.H.; project administration, C.S-R.; funding acquisition, D.R.

Funding: This research was funded by the National Institute of Environmental Health Sciences grant numbers 5 R42 ES026908 03 and 1R41ES032435-01, and the National Institute of General Medical Sciences grant number 1R43GM139439-01 (DSR).

Acknowledgments: Authors thank Yudan Li, Runrui Dang, and Xuechun Qi for contributions to some of the data, Dr. Seema Agarwal (Georgetown University Lombardi Cancer Center) for help with the conditional reprogramming of the CD133(+) BAKP cells to derive the BAKR cell line, and Dr. Karen Creswell (Georgetown University Medical Center Flow Cytometry core facility) for help with flow cytometry analysis.

Conflicts of Interest: The authors declare no conflict of interest.

\section{References}

1. SEER Surveillance, E., and End Results Program, Cancer Stat Facts: Melanoma of the Skin. Bethesda, MD, https://seer.cancer.gov/statfacts/html/melan.html,, 2021. posted to the SEER web site as of May 2021.

2. Paluncic, J., et al., Roads to melanoma: Key pathways and emerging players in melanoma progression and oncogenic signaling. Biochim Biophys Acta, 2016. 1863(4): p. 770-84. 
3. Spagnolo, F., et al., Survival of patients with metastatic melanoma and brain metastases in the era of MAP-kinase inhibitors and immunologic checkpoint blockade antibodies: A systematic review. Cancer Treat Rev, 2016. 45: p. 38-45.

4. Ugurel, S., et al., Survival of patients with advanced metastatic melanoma: the impact of novel therapies-update 2017. Eur J Cancer, 2017. 83: p. 247-257.

5. Sandri, S., et al., Vemurafenib resistance increases melanoma invasiveness and modulates the tumor microenvironment by MMP-2 upregulation. Pharmacol Res, 2016. 111: p. 523-533.

6. Kalal, B.S., D. Upadhya, and V.R. Pai, Chemotherapy Resistance Mechanisms in Advanced Skin Cancer. Oncol Rev, 2017. 11(1): p. 326.

7. $\quad$ Berger, M.F., et al., Melanoma genome sequencing reveals frequent PREX2 mutations. Nature, 2012. 485: p. 502.

8. Pleasance, E.D., et al., A comprehensive catalogue of somatic mutations from a human cancer genome. Nature, 2009. 463 : p. 191.

9. Wang, E., et al., Clonal persistence and evolution during a decade of recurrent melanoma. J Invest Dermatol, 2006. $126(6)$ : p. $1372-7$.

10. Bhatia, S., S.S. Tykodi, and J.A. Thompson, Treatment of metastatic melanoma: an overview. Oncology (Williston Park), 2009. 23(6): p. 488-96.

11. Giampietri, C., et al., Lipid Storage and Autophagy in Melanoma Cancer Cells. Int J Mol Sci, 2017. 18(6).

12. Schatton, T., et al., Identification of cells initiating human melanomas. Nature, 2008. 451(7176): p. 345-349.

13. Sharma, B.K., et al., Clonal dominance of CD133+ subset population as risk factor in tumor progression and disease recurrence of human cutaneous melanoma. Int J Oncol, 2012. 41(5): p. 1570-6.

14. Fang, D., et al., A tumorigenic subpopulation with stem cell properties in melanomas. Cancer Res, 2005. 65(20): p. 9328-37.

15. Thapa, R. and G.D. Wilson, The Importance of CD44 as a Stem Cell Biomarker and Therapeutic Target in Cancer. Stem Cells Int, 2016. 2016: p. 2087204.

16. Hendrix, M.J., et al., Molecular plasticity of human melanoma cells. Oncogene, 2003. 22(20): p. 3070-5.

17. Klein, W.M., et al., Increased expression of stem cell markers in malignant melanoma. Modern Pathology, 2007. 20(1): p. $102-107$.

18. Civenni, G., et al., Human CD271-positive melanoma stem cells associated with metastasis establish tumor heterogeneity and long-term growth. Cancer Res, 2011. 71(8): p. 3098-109.

19. Luo, Y., et al., ALDH1A isozymes are markers of human melanoma stem cells and potential therapeutic targets. Stem Cells, 2012. 30(10): p. 2100-13.

20. Akiyama, M., et al., Inhibition of the stem cell marker nestin reduces tumor growth and invasion of malignant melanoma. J Invest Dermatol, 2013. 133(5): p. 1384-7.

21. Frank, N.Y., et al., VEGFR-1 expressed by malignant melanoma-initiating cells is required for tumor growth. Cancer Res, 2011. 71(4): p. $1474-85$.

22. Roesch, A., et al., A temporarily distinct subpopulation of slow-cycling melanoma cells is required for continuous tumor growth. Cell, 2010. 141(4): p. 583-94.

23. Sanai, N., A. Alvarez-Buylla, and M.S. Berger, Neural stem cells and the origin of gliomas. N Engl J Med, 2005. $353(8):$ p. 811-22.

24. Yin, A.H., et al., AC133, a novel marker for human hematopoietic stem and progenitor cells. Blood, 1997. 90(12): p. 5002-12.

25. Lai, G.M., et al., Active fraction of Taiwanofungus camphoratus (HS7) exerted anticancer effects through multiple molecule targeting and elimination of cancer stem-like cells in lung cancer and hepatoma cells. Cancer Research, 2009. 69.

26. Mizrak, D., M. Brittan, and M. Alison, CD133: molecule of the moment. J Pathol, 2008. 214(1): p. 3-9.

27. Shmelkov, S.V., et al., AC133/CD133/Prominin-1. Int J Biochem Cell Biol, 2005. 37(4): p. 715-9.

28. Dowland, S.N., et al., Prominin-1 glycosylation changes throughout early pregnancy in uterine epithelial cells under the influence of maternal ovarian hormones. Reprod Fertil Dev, 2017. 29(6): p. 1194-1208.

29. Collins, A.T., et al., Prospective identification of tumorigenic prostate cancer stem cells. Cancer Res, 2005. 65(23): p. $10946-51$.

30. Ferrandina, G., et al., Expression of CD133-1 and CD133-2 in ovarian cancer. Int J Gynecol Cancer, 2008. 18(3): p. 506-14.

31. Hermann, P.C., et al., Distinct populations of cancer stem cells determine tumor growth and metastatic activity in human pancreatic cancer. Cell Stem Cell, 2007. 1(3): p. 313-23.

32. Kim, Y.S., et al., Cancer Stem Cell Molecular Markers Verified in vivo. Biochemistry Moscow-Supplement Series B-Biomedical Chemistry, 2017. 11(1): p. 43-54.

33. O'Brien, C.A., et al., A human colon cancer cell capable of initiating tumour growth in immunodeficient mice. Nature, 2007. 445(7123): p. 106-10.

34. Ricci-Vitiani, L., et al., Identification and expansion of human colon-cancer-initiating cells. Nature, 2007. 445(7123): p. 111-5.

35. Singh, S.K., et al., Identification of a cancer stem cell in human brain tumors. Cancer Res, 2003. 63(18): p. 5821-8.

36. Singh, S.K., et al., Identification of human brain tumour initiating cells. Nature, 2004. 432(7015): p. 396-401.

37. Suetsugu, A., et al., Characterization of CD133+ hepatocellular carcinoma cells as cancer stem/progenitor cells. Biochem Biophys Res Commun, 2006. 351(4): p. 820-4.

38. Galli, R., et al., Isolation and characterization of tumorigenic, stem-like neural precursors from human glioblastoma. Cancer Res, 2004. 64(19): p. 7011-21.

39. Quintana, E., et al., Efficient tumour formation by single human melanoma cells. Nature, 2008. 456(7222): p. 593-8.

40. Hendrix, M.J., et al., Reprogramming metastatic tumour cells with embryonic microenvironments. Nat Rev Cancer, 2007. 7(4): p. 24655.

41. Kirschmann, D.A., et al., Molecular pathways: vasculogenic mimicry in tumor cells: diagnostic and therapeutic implications. Clin Cancer Res, 2012. 18(10): p. 2726-32. 
42. Mihic-Probst, D., et al., Tumor cell plasticity and angiogenesis in human melanomas. PLoS One, 2012. 7(3): p. e33571.

43. Simbulan-Rosenthal, C.M., et al., CRISPR-Cas9 Knockdown and Induced Expression of CD133 Reveal Essential Roles in Melanoma Invasion and Metastasis. Cancers (Basel), 2019. 11(10).

44. Angelastro, J.M. and M.W. Lame, Overexpression of CD133 promotes drug resistance in C6 glioma cells. Mol Cancer Res, 2010. 8(8): p. 1105-15.

45. Guo, J.C., et al., microRNA-448 inhibits stemness maintenance and self-renewal of hepatocellular carcinoma stem cells through the MAGEA6-mediated AMPK signaling pathway. J Cell Physiol, 2019.

46. Miyabayashi, T., et al., Vaccination with CD133(+) melanoma induces specific Th17 and Th1 cell-mediated antitumor reactivity against parental tumor. Cancer Immunol Immunother, 2011. 60(11): p. 1597-608.

47. Ma, S., et al., CD133+ HCC cancer stem cells confer chemoresistance by preferential expression of the Akt/PKB survival pathway. Oncogene, 2008. 27(12): p. 1749-58.

48. Song, S., et al., Interaction between CD133 and PI3K-p85 promotes chemoresistance in gastric cancer cells. Am J Transl Res, 2018. 10(1): p. 304-314.

49. Wei, Y., et al., Activation of PI3K/Akt pathway by CD133-p85 interaction promotes tumorigenic capacity of glioma stem cells. Proc Natl Acad Sci U S A, 2013. 110(17): p. 6829-34.

50. Datta, S.R., et al., Akt phosphorylation of BAD couples survival signals to the cell-intrinsic death machinery. Cell, 1997. 91(2): p. 231-41.

51. Tewari, M., et al., Yama/CPP32 beta, a mammalian homolog of CED-3, is a CrmA-inhibitable protease that cleaves the death substrate poly(ADP-ribose) polymerase. Cell, 1995. 81(5): p. 801-9.

52. Cuvillier, O., et al., Sphingosine 1-phosphate inhibits activation of caspases that cleave poly(ADP-ribose) polymerase and lamins during Fas- and ceramide-mediated apoptosis in Jurkat T lymphocytes. J Biol Chem, 1998. 273(5): p. 2910-6.

53. Rosenthal, D.S., et al., Intact cell evidence for the early synthesis, and subsequent late apopain-mediated suppression, of poly(ADP-ribose) during apoptosis. Exp Cell Res, 1997. 232(2): p. 313-21.

54. Simbulan-Rosenthal, C.M., et al., CD133 Is Associated with Increased Melanoma Cell Survival after Multikinase Inhibition. Journal of Oncology, 2019. 2019: p. 19.

55. Tse, C., et al., ABT-263: a potent and orally bioavailable Bcl-2 family inhibitor. Cancer Res, 2008. 68(9): p. 3421-8.

56. Frederick, D.T., et al., Clinical profiling of BCL-2 family members in the setting of BRAF inhibition offers a rationale for targeting de novo resistance using BH3 mimetics. PLoS One, 2014. 9(7): p. e101286.

57. Amaral, T., et al., An open-label, single-arm, phase II trial of buparlisib in patients with melanoma brain metastases not eligible for surgery or radiosurgery-the BUMPER study. Neurooncol Adv, 2020. 2(1): p. vdaa140.

58. Huang, H., et al., Costunolide suppresses melanoma growth via the AKT/mTOR pathway in vitro and in vivo. Am J Cancer Res, 2021. 11(4): p. 1410-1427.

59. Hwang, S.Y., et al., Alternative Options for Skin Cancer Therapy via Regulation of AKT and Related Signaling Pathways. Int J Mol Sci, 2020. 21(18).

60. Schmohl, J.U., et al., Heterodimeric Bispecific Single Chain Variable Fragments (scFv) Killer Engagers (BiKEs) Enhance NK-cell Activity Against CD133+ Colorectal Cancer Cells. Target Oncol, 2016. 11(3): p. 353-61.

61. Swaminathan, S.K., et al., Identification of a novel monoclonal antibody recognizing CD133. J Immunol Methods, 2010. 361(1-2): p. $110-5$.

62. Waldron, N.N., et al., Targeting tumor-initiating cancer cells with dCD133KDEL shows impressive tumor reductions in a xenotransplant model of human head and neck cancer. Mol Cancer Ther, 2011. 10(10): p. 1829-38.

63. Alibolandi, M., et al., CD133-targeted delivery of self-assembled PEGylated carboxymethylcellulose-SN38 nanoparticles to colorectal cancer. Artif Cells Nanomed Biotechnol, 2018. 46(sup1): p. 1159-1169.

64. DiVito, K.A., et al., Id2, Id3 and Id4 overcome a Smad7-mediated block in tumorigenesis, generating TGF-beta-independent melanoma. Carcinogenesis, 2014. 35(4): p. 951-8.

65. DiVito, K.A., et al., Smad7 restricts melanoma invasion by restoring N-cadherin expression and establishing heterotypic cell-cell interactions in vivo. Pigment Cell Melanoma Res, 2010. 23(6): p. 795-808.

66. Falzone, L., et al., Computational identification of microRNAs associated to both epithelial to mesenchymal transition and NGAL/MMP9 pathways in bladder cancer. Oncotarget, 2016. 7(45): p. 72758-72766.

67. Yang, L., et al., Tumor suppressor microRNA-34a inhibits cell migration and invasion by targeting MMP-2/MMP-9/FNDC3B in esophageal squamous cell carcinoma. Int J Oncol, 2017. 51(1): p. 378-388.

68. Simbulan-Rosenthal, C.M., et al., Inorganic polyphosphates are important for cell survival and motility of human skin keratinocytes. Exp Dermatol, 2015. 24(8): p. 636-9.

69. Simbulan-Rosenthal, C.M., et al., The repurposed anthelmintic mebendazole in combination with trametinib suppresses refractory NRASQ61K melanoma. Oncotarget, 2017. 8(8): p. 12576-12595. 